\title{
The viscosity solutions approach to swing options pricing under a regime-switching mean-reverting model
}

\author{
Lingjie Shao ${ }^{1 *}$ (D), Kaili Xiang ${ }^{1}$ and Yang Song'
}

\section{"Correspondence:}

ljshao@2014.swufe.edu.cn

${ }^{1}$ School of Economics and

Mathematics, Southwestern

University of Finance and

Economics, Chengdu, China

\begin{abstract}
In this paper, we study the valuation of swing options on electricity markets with local volume and refraction time constraints, under the setting that the dynamic of the underlying spot price is a 2 -state regime-switching mean-reverting process. We derive the corresponding optimal multiple stopping problem, reduce it to a sequence of optimal single stopping problems, and further find that those value functions satisfy HJB variational inequalities subject to suitable conditions. Then after a prior estimation for the value functions, the viscosity solutions approach is adopted to get existence and uniqueness results in viscosity sense.
\end{abstract}

Keywords: Swing options; Regime-switching; Mean-reverting; Variational inequalities; Viscosity solutions

\section{Introduction}

In real electricity markets, swing options provide the holder with rights to exercise repeatedly under certain constraints, such as limits on the local volume, the global volume, and refraction time etc. However, those trading rules add complexity to the associated valuation, which can be handled as stochastic optimal control problems. Besides the specification of option contracts, the other one of fundamental settings of the whole valuation procedure is the dynamic process characterizing the behavior of electricity prices, which remain high for a period of time after a jump, according to the empirical researches like [14] and [5]. Among various kinds of models, the regime-switching type is widely adopted. Wahab et al. [6] investigate the valuation problem under a three-state regime-switching model, where one regime is the Ornstein-Uhlenbeck type, and another two are Gaussian processes with constant coefficients. Wahab et al. [7] deal with a $n$-state regime-switching model where processes in all regime are geometric Brownian motion. Chiarella et al. [8] handle the valuation problem under a regime-switching forward curve model, and Bauerle et al. [9] choose a 2-state regime-switching model.

In all of [6-9], the lattice method is applied to price swing options, though with different choices of underlying price models. The lattice method concentrates on how to find the value function (a specific type of conditional expectation) by directly computing expectation iteratively with lattice. More specifically, in the light of stochastic control theory, the dynamic programming principle is satisfied by the value function leads us to the Bellman

(c) The Author(s) 2018. This article is distributed under the terms of the Creative Commons Attribution 4.0 International License (http://creativecommons.org/licenses/by/4.0/), which permits unrestricted use, distribution, and reproduction in any medium, provided you give appropriate credit to the original author(s) and the source, provide a link to the Creative Commons license, and indicate if changes were made. 
equation. Then lattices in different form can be applied to dealing with the conditional expectations arising in the corresponding Bellman's equations. Different from the lattice method, another approach to tackling this problem is to seek the PDE characterization of the value function, which leads to the HJB variational inequalities. In this direction, Dahlgren et al. in [10] and [11] derive the variational inequalities and solve them by the finite difference method. In addition, basing on [12], Wilhelm and Winter [13] solve the related variational inequalities by finite element method. Besides, according to [14] and [15], the viscosity solutions approach is a powerful tool to deal with stochastic optimal control problems, and it is well known that the value function is the unique viscosity solution to the HJB-type equation or variational inequality subject to certain conditions, and that this approach can also help studying properties (e.g. convergence and stability) of numerical schemes. For pricing swings, the viscosity solution approach has been used in [16] and [17].

In this paper, the electricity price model we use is of a regime-switching mean-reverting type with two states, where the mean-reverting characteristic is added to the model adopted in [6], and this modification is motivated by empirical researches in [1] and [18], which report that electricity spot price is equipped with the mean-reversion feature. As for the swing option contract, we assume that there exist: (i) the local volume constraint, that is, the holder can either exercise one volume of spot or none each time she uses one right, and (ii) the refraction time constraint, which requires a fixed time break between two successive exercises. Under those settings, we formulate the pricing of the swing option as an optimal multiple stopping problem. Basing on the work of Carmona and Touzi [12], we reduce this problem. After the reduction, continuity and boundedness of value functions is obtained and then we try to characterize the value functions as the unique viscosity solution of the associated HJB variational inequalities. First, existence results can be received. In addition, we prove the comparison principle fitting this problem which ensure the uniqueness of the solutions in viscosity sense.

The structure of this paper is as follows. Section 2 introduces the model for the underlying electricity price and derives the variational inequalities that the value functions formally satisfy. Section 3 treats the properties of the value functions. Section 4 characterizes the value functions as viscosity solutions of the associated variational inequalities. Section 5 gives the comparison principle and the uniqueness result.

\section{Formulation of the problem}

\subsection{Mean-reverting regime-switching model and swing options}

For a fixed maturity $T$, we work on a probability space $\left(\Omega, \mathcal{F},\{\mathcal{F}(t)\}_{t \in[0, T]}, \mathbb{Q}\right)$ and a real

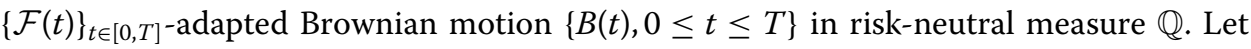
$\{\alpha(t)\}_{0 \leq t \leq T}$ be an independent time-homogeneous Markov chain with values in a finite state space $\mathcal{M}:=\{1,2\}$, and the related generator matrix $\mathbf{P}=\left(q_{i j}\right)_{2 \times 2}$, satisfying $q_{i j} \geq 0$ and $\sum_{j=1}^{2} q_{i j}=0$ for each $i, j \in \mathcal{M}$ and $i \neq j$.

We model the electricity price by the stochastic process $\{S(t)\}_{t \in[0, T]}$, which is governed by the following:

$$
d S(t)=\beta(\alpha(t))(\xi(\alpha(t))-S(t)) d t+\sigma(\alpha(t)) d B^{\mathbb{Q}}(t) ; \quad S(0)=S_{0}, \quad \alpha(0)=i
$$

where $S_{0}$ and $i$ are initial data; $\beta(i), \xi(i)$ and $\sigma(i)$ for each $i \in \mathcal{M}$ are constants representing the speed of adjustment, the long-term mean level, and the volatility. Moreover, we notice 
that the usual Lipschitz and linear growth conditions are satisfied to ensure the existence and uniqueness of the strong solution to the $\operatorname{SDE}(1)$.

On the settings of swings, we follow [12]. Consider a swing option with $n \in \mathbb{N}^{+}$exercise rights during the time period $[0, T]$. The strike price is $K \in \mathbb{R}^{+}$, thus the holder gets the payoff $\phi(x)=(K-x)^{+}$. All of the above parameters are fixed. And there are two rules the holder must obey:

1 Each time the holder use the right, she can exercise either one volume or none.

2 If one right is used at time $t$, the holder cannot use another right until after the time $t+\delta$, where $\delta>0$ is the refraction period.

We call the first item above the local volume constraint and the second the refraction time constraint.

\subsection{Variational inequalities of value functions}

In this subsection, we start from introducing the value function of this problem. For simplicity, we sometimes use $f_{i}(t, s)$ to stand for any function $f(t, s, i):[0, T] \times \mathbb{R}^{+} \times \mathcal{M} \mapsto \mathbb{R}$.

Definition 1 Let $(t, s, i) \in[0, T] \times \mathbb{R}^{+} \times \mathcal{M}$. For fixed integer $n \in \mathbb{N}$, we define the value function

$$
V_{i}^{(n)}(t, s):=\sup _{\vec{\tau} \in \mathcal{T}_{t, T}^{(n)}} \mathbb{E}\left[\sum_{k=1}^{n} e^{-r\left(\tau_{k}-t\right)} \phi\left(S\left(\tau_{k}\right)\right) \mid S_{t}=s, \alpha_{t}=i\right],
$$

where $\mathcal{T}_{t, T}^{(n)}$ is the collection of $\vec{\tau}:=\left(\tau_{1}, \tau_{2}, \ldots, \tau_{n}\right)$ having the following properties:

- $t \leq \tau_{1} \leq T$ a.s.,

- $\tau_{i}-\tau_{i-1} \geq \delta$ on $\left\{0 \leq \tau_{i-1} \leq T\right\}$ a.s., $\forall i=1,2, \ldots, n$,

- $\tau_{i}:=T+$ on $\left\{\tau_{i}>T\right\}$ a.s., $\forall i=1,2, \ldots, n$;

moreover, $T+>T$ is a cemetery time and we set $\phi\left(S_{T_{+}}\right) \equiv 0$.

Here, the notation $V_{i}^{(n)}(t, s)$ denotes the value of swing option at time $t$ with state value $(S(t), \alpha(t))=(s, i)$, and the superscript $n$ is used to emphasize that the holder still has $n$ rights before the expiry.

For the above value functions, we state in the following proposition an iterative equation showing the relationship between $V^{(n)}$ and $V^{(n-1)}$. It is this relationship that transforms the optimal multiple stopping problem into a sequence of optimal single stopping problems.

Proposition 1 Let $(t, s, i) \in[0, T] \times \mathbb{R}^{+} \times \mathcal{M}$. For fixed integer $n \in \mathbb{N}$, the value function $V_{i}^{(n)}$ of (2) can be equivalently written follows.

(i) For $n=0$,

$$
V_{i}^{(0)}(t, s)=0
$$

(ii) For $n \geq 1$,

$$
V_{i}^{(n)}(t, s)=\sup _{\tau \in \mathcal{T}_{t, T}} E^{\mathbb{Q}}\left[e^{-r(\tau-t)} G_{\alpha(\tau)}^{(n)}(\tau, S(\tau)) \mid S(t)=s, \alpha(t)=i\right]
$$


where $\mathcal{T}_{t, T}$ is the same as in the above definition, and

$$
G_{i}^{(n)}(t, s):=\left\{\begin{array}{l}
(K-s)^{+}+E^{\mathbb{Q}}\left[e^{-r \delta} V_{\alpha(t+\delta)}^{(n-1)}(t+\delta, S(t+\delta)) \mid S(t)=s, \alpha(t)=i\right] \\
t \in[0, T-\delta] \\
(K-s)^{+} \\
t \in(T-\delta, T]
\end{array}\right.
$$

where $\delta$ is the refraction time.

Proof Notice that the reward (or payoff) process $\phi\left(S_{t}\right)=\left(K-S_{t}\right)^{+}$has continuous paths and the strong Markov property, which indicates that it satisfies conditions (2.1), (2.2) and (2.8) in [12]. In addition, according to [19] we find that the filtration $\{\mathcal{F}(t)\}_{t \in[0, T]}$ satisfies the usual conditions and additional requirements (2.10) in [12]. In this way, we can apply Theorem 1 in [12] to rewrite the problem (2) into the iteration form (4) with (5).

Remark 1 The specific form of $G_{i}^{(n)}$ of (5) in Proposition 1 can be further written as follows.

(i) For $n=1$,

$$
G_{i}^{(1)}(t, s)=(K-s)^{+}, \quad \forall t \in[0, T]
$$

(ii) For $n \geq 2, G_{i}^{(n)}$ involves a European option price, i.e.,

$$
G_{i}^{(n)}(t, s):= \begin{cases}(K-s)^{+}+f_{i}^{(n)}(0, s), & t \in[0, T-\delta], \\ (K-s)^{+}, & t \in(T-\delta, T],\end{cases}
$$

where $\left(f_{1}^{(n)}(\tau, s), f_{2}^{(n)}(\tau, s)\right)$ satisfies

$$
\left\{\begin{array}{l}
\mathcal{L}_{1} f_{1}^{(n)}+q_{12}\left(f_{2}^{(n)}-f_{1}^{(n)}\right)=0, \quad \tau \in[0, \delta), s>0, \\
\mathcal{L}_{2} f_{2}^{(n)}+q_{21}\left(f_{1}^{(n)}-f_{2}^{(n)}\right)=0, \quad \tau \in[0, \delta), s>0, \\
f_{1}^{(n)}(\delta, \cdot)=f_{2}^{(n)}(\delta, \cdot)=\sum_{j=1}^{2} w_{i j}(\delta) V_{j}^{(n-1)}(t+\delta, \cdot), \quad s>0
\end{array}\right.
$$

in which

$$
\mathcal{L}_{i} V_{i}^{(n)}:=\frac{\partial}{\partial t} V_{i}^{(n)}+\frac{1}{2} \sigma^{2}(i) \frac{\partial^{2}}{\partial s^{2}} V_{i}^{(n)}+\beta(i)(\xi(i)-s) \frac{\partial}{\partial s} V_{i}^{(n)}-r V_{i}^{(n)}, \quad i \in \mathcal{M} .
$$

To see how (6) comes about, we define

$$
\begin{array}{rl}
u_{i}^{(n)}(t, s):= & E^{\mathbb{Q}}\left[e^{-r \delta} \sum_{j=1}^{2} w_{i j}(\delta) V_{j}^{(n-1)}(t+\delta, S(t+\delta)) \mid S(t)=s, \alpha(t)=i\right], \\
t & t[0, T-\delta], s \in \mathbb{R}^{+}, i=1,2 .
\end{array}
$$

Let $f_{i}^{(n)}(0, s)=u_{i}^{(n)}(t, s)$, then using the Feynman-Kac formula, one gets what one wants.

Having transformed the problem in Proposition 1, now we show the PDE characterization of the value functions $V_{i}^{(n)}$ in the form of variational inequalities. 
Theorem 1 For $n \in N^{+}$, the pair $\left(V_{1}^{(n)}(t, s), V_{2}^{(n)}(t, s)\right)$ formally satisfy the coupled variational inequalities

$$
\begin{cases}\min \left\{-\mathcal{L}_{1} V_{1}^{(n)}-q_{12}\left(V_{2}^{(n)}-V_{1}^{(n)}\right), V_{1}^{(n)}-G_{1}^{(n)}\right\}=0, & s>0,0<t<T, \\ \min \left\{-\mathcal{L}_{2} V_{2}^{(n)}-q_{21}\left(V_{1}^{(n)}-V_{2}^{(n)}\right), V_{2}^{(n)}-G_{2}^{(n)}\right\}=0, & s>0,0<t<T, \\ V_{1}^{(n)}=V_{2}^{(n)}=(K-s)^{+}, \quad s>0, t=T, & \end{cases}
$$

where $\mathcal{L}_{i} V_{i}^{(n)}$ and $G_{i}^{(n)}$ are the same as in Proposition 1.

Proof Let $\zeta:[0, T] \times \mathbb{R}^{+} \times \mathcal{M} \mapsto \mathbb{R}$ be smooth function. Denote $\zeta_{i}(t, s):=\zeta(t, s, i)$. Let $\tau$ be a stopping time in $\mathcal{T}_{t, T}$. Then we have from the Dynkin formula that

$$
\begin{aligned}
e^{-r(\tau-t)} \zeta(\tau, S(\tau), \alpha(\tau))-\zeta(t, S(t), \alpha(t)) \\
\quad=\int_{t}^{\tau} d\left(e^{-r(\theta-t)} \zeta(\theta, S(\theta), \alpha(\theta))\right) \\
\quad=\int_{t}^{\tau}\left[e^{-r(\theta-t)}\left(\mathcal{L}_{\alpha(\theta)} \zeta_{\alpha(\theta)}+q_{i 1}\left(\zeta_{1}-\zeta_{2}\right)\right) d \theta+e^{-r(\theta-t)} d M(\theta)\right],
\end{aligned}
$$

where $M$ is a martingale under measure $\mathbb{Q}$, and

$$
\mathcal{L}_{i} \zeta_{i}:=\frac{\partial}{\partial t} \zeta_{i}+\frac{1}{2} \sigma^{2}(i) \frac{\partial^{2}}{\partial s^{2}} \zeta_{i}+\beta(i)(\xi(i)-s) \frac{\partial}{\partial s} \zeta_{i}-r \zeta_{i}, \quad i \in \mathcal{M}
$$

Taking the expectation on both sides, we obtain

$$
\begin{aligned}
\zeta_{i}(t, s) & \\
= & E^{\mathbb{Q}}\left[e^{-r(\tau-t)} \zeta_{\alpha(\tau)}(\tau, S(\tau))\right. \\
& \left.-\int_{t}^{\tau} e^{-r(\theta-t)}\left(\mathcal{L}_{\alpha(\theta)} \zeta_{\alpha(\theta)}+q_{i j}\left(\zeta_{j}(\theta, S(\theta))-\zeta_{i}(\theta, S(\theta))\right)\right) d \theta \mid S(t)=s, \alpha(t)=i\right],
\end{aligned}
$$

where $i, j \in \mathcal{M}, j \neq i$. Then under the assumption that

$$
\zeta_{i}(\cdot, \cdot) \geq G_{i}^{(n)}(\cdot, \cdot) \quad \text { and } \quad-\mathcal{L}_{i} \zeta_{i}(\cdot, \cdot)-q_{i j}\left(\zeta_{j}(\cdot, \cdot)-\zeta_{i}(\cdot, \cdot)\right) \geq 0, \quad i \in \mathcal{M}
$$

we have

$$
\zeta_{i}(t, s) \geq E^{\mathbb{Q}}\left[e^{-r(\tau-t)} G_{\alpha(\tau)}^{(n)}(\tau, S(\tau)) \mid S(t)=s, \alpha(t)=i\right], \quad i \in \mathcal{M},
$$

and by taking the supreme on both sides, it follows that

$$
\zeta_{i}(t, s) \geq V_{i}^{(n)}(t, s)
$$

Hence, we assume that $\left(\zeta_{1}(t, s), \zeta_{2}(t, s)\right)$ is the solution of

$$
\left\{\begin{array}{l}
\min \left\{-\mathcal{L}_{i} \zeta_{i}-q_{i j}\left(\zeta_{j}-\zeta_{i}\right), \zeta_{i}-G_{i}^{(n)}\right\}=0, \quad 0<t<T, s>0, i, j \in \mathcal{M}, j \neq i \\
\zeta_{i}=G_{i}^{(n)}(s, i, T)=(K-s)^{+}, \quad t=T, s>0, i \in \mathcal{M}
\end{array}\right.
$$


On the other hand, define

$$
\tau^{*}:=\inf \left\{t \leq \tau \leq T \mid \zeta_{\alpha(\tau)}^{(n)}\left(\tau, S_{\tau}\right)=G^{(n)}(\tau, S(\tau), \alpha(\tau))\right\} \in \mathcal{T}_{t, T}
$$

which implies that

$$
\begin{aligned}
\zeta_{i}(t, s)= & E^{\mathbb{Q}}\left[e^{-r\left(\tau^{*}-t\right)} \zeta_{\alpha\left(\tau^{*}\right)}\left(\tau^{*}, S\left(\tau^{*}\right)\right)\right. \\
& -\int_{t}^{\tau^{*}} e^{-r(\theta-t)}\left(\mathcal{L}_{\alpha(\theta)} \zeta_{\alpha(\theta)}+q_{i j}\left(\zeta_{j}-\zeta_{i}\right) d \theta \mid S(t)=s, \alpha(t)=i\right] \\
= & E^{\mathbb{Q}}\left[e^{-r\left(\tau^{*}-t\right)} G_{\alpha\left(\tau^{*}\right)}^{(n)}\left(\tau^{*}, S\left(\tau^{*}\right)\right) \mid S(t)=s, \alpha(t)=i\right] \leq V_{i}^{(n)}(t, s) .
\end{aligned}
$$

Above all, if $V^{(n)}$ is as smooth as $\zeta$, then it is the solution of the problem (7).

\subsection{Viscosity solutions}

In this subsection, after introducing the notion of viscosity solution, we state that the value functions in this paper is the unique viscosity solution of the problem (7).

Definition 2 (Viscosity supersolution) For $(t, s) \in[0, T] \times \mathbb{R}^{+}, i \in \mathcal{M}$ and $n \in N^{+}$, assume that $f_{i}^{(n)}(t, s)$ satisfies the following four conditions:

$1 f_{i}^{(n)}(t, s)$ is locally bounded.

$2 f_{i}^{(n)}(\cdot, \cdot)$ is continuous in $[0, T] \times \mathbb{R}^{+}$.

$3 f_{1}^{(n)}(T, s)=f_{2}^{(n)}(T, s)=(K-s)^{+}$on $\{T\} \times \mathbb{R}^{+}$.

4. For $\forall(\bar{t}, \bar{s}) \in[0, T) \times \mathbb{R}^{+}$and $\forall \phi \in C^{1,2}\left([0, T) \times \mathbb{R}^{+}\right)$such that

$$
0=f_{i}^{(n)}(\bar{t}, \bar{s})-\phi(\bar{t}, \bar{s})=\text { strict } \min _{[0, T) \times \mathbb{R}^{+}}\left(f_{i}^{(n)}(t, s)-\phi(t, s)\right)
$$

we always have

$$
\begin{aligned}
& \min \left\{-\frac{\partial \phi(\bar{t}, \bar{s})}{\partial t}-\frac{1}{2} \sigma^{2}(i) \frac{\partial^{2} \phi(\bar{t}, \bar{s})}{\partial s^{2}}-\beta(i)(\xi(i)-\bar{s}) \frac{\partial \phi(\bar{t}, \bar{s})}{\partial s}\right. \\
& \left.\quad+r \phi(\bar{t}, \bar{s}, i)-q_{i j}\left(f_{j}^{(n)}(\bar{t}, \bar{s})-\phi(\bar{t}, \bar{s})\right), \phi(\bar{t}, \bar{s})-G_{i}^{(n)}(\bar{t}, \bar{s})\right\} \geq 0, \\
& \quad i, j \in \mathcal{M}, j \neq i,
\end{aligned}
$$

where

$$
G_{i}^{(n)}(\bar{t}, \bar{s}):=\left\{\begin{array}{l}
(K-\bar{s})^{+}+E^{\mathbb{Q}}\left[e^{-r \delta} f_{\alpha(\bar{t}+\delta)}^{(n-1)}(\bar{t}+\delta, S(\bar{t}+\delta)) \mid S(\bar{t})=\bar{s}, \alpha(\bar{t})=i\right], \\
\quad \bar{t} \in[0, T-\delta], \\
(K-\bar{s})^{+} \\
\quad \bar{t} \in(T-\delta, T) .
\end{array}\right.
$$

Then we say $f_{i}^{(n)}(t, s)$ is a continuous viscosity supersolution of the variational inequalities in (7) on $[0, T) \times \mathbb{R}^{+}$. 
Definition 3 (Viscosity subsolution) For $(t, s) \in[0, T] \times \mathbb{R}^{+}, i \in \mathcal{M}$ and $n \in N^{+}$, assume that $f_{i}^{(n)}(t, s)$ satisfies the following four conditions:

$1 f_{i}^{(n)}(t, s)$ is locally bounded.

$2 f_{i}^{(n)}(\cdot, \cdot)$ is continuous in $[0, T] \times \mathbb{R}^{+}$.

$3 f_{1}^{(n)}(T, s)=f_{2}^{(n)}(T, s)=(K-s)^{+}$on $\{T\} \times \mathbb{R}^{+}$.

4. For $\forall(\bar{t}, \bar{s}) \in[0, T) \times \mathbb{R}^{+}$and $\forall \phi \in C^{1,2}\left([0, T) \times \mathbb{R}^{+}\right)$such that

$$
0=f_{i}^{(n)}(\bar{t}, \bar{s})-\phi(\bar{t}, \bar{s})=\text { strict } \max _{[0, T) \times \mathbb{R}^{+}}\left(f_{i}^{(n)}(t, s)-\phi(t, s)\right),
$$

we always have

$$
\begin{aligned}
& \min \left\{-\frac{\partial \phi(\bar{t}, \bar{s})}{\partial t}-\frac{1}{2} \sigma^{2}(i) \frac{\partial^{2} \phi(\bar{t}, \bar{s})}{\partial s^{2}}-\beta(i)(\xi(i)-\bar{s}) \frac{\partial \phi(\bar{t}, \bar{s})}{\partial s}\right. \\
& \left.+r \phi(\bar{t}, \bar{s}, i)-q_{i j}\left(f_{j}^{(n)}(\bar{t}, \bar{s})-\phi(\bar{t}, \bar{s})\right), \phi(\bar{t}, \bar{s})-G_{i}^{(n)}(\bar{t}, \bar{s})\right\} \leq 0, \\
& \quad i, j \in \mathcal{M}, j \neq i,
\end{aligned}
$$

where $G_{i}^{(n)}(\bar{t}, \bar{s})$ is the same as in (8).

Then we say $f_{i}^{(n)}(t, s)$ is a continuous viscosity subsolution of the variational inequalities in (7) on $[0, T) \times \mathbb{R}^{+}$.

Definition 4 (Viscosity solution) For $(t, s) \in[0, T] \times \mathbb{R}^{+}, i \in \mathcal{M}$ and $n \in N^{+}$, we say that $f_{i}^{(n)}(t, s)$ is a continuous viscosity solution of $(7)$ on $[0, T] \times \mathbb{R}^{+}$if it is both a continuous supersolution and a continuous subsolution of the variational inequalities in (7).

Theorem 2 For $(t, s) \in[0, T] \times \mathbb{R}^{+}, i \in \mathcal{M}$ and $n \in N^{+}, V_{i}^{(n)}(t, s)$ defined in Definition 1 is the unique viscosity solution of the problem (7).

In Sects. 4 and 5, we will show the proofs of the existence and uniqueness results, respectively.

\section{Properties of the value function}

In this part, we derive properties including continuity and local boundedness of the value function to demonstrate that it satisfies the first two conditions in Definition 2 (or in Definition 3, notice that they are the same).

Lemma 1 Let $(t, s) \in[0, T] \times \mathbb{R}^{+}$, then, for each $i \in \mathcal{M}$ and $n \in N^{+}$, the value function $V_{i}^{(n)}(t, s)$ is continuous with respect to $s$.

Proof For each $i \in \mathcal{M}$, given $s_{1}, s_{2}$ and $t \in[0, T]$, let $S_{1}$ and $S_{2}$ be two solutions of the SDE (1) with $S_{1}(t)=s_{1}$ and $S_{2}(t)=s_{2}$, respectively. We adopt mathematical induction.

(i) For $n=1$, we have

$$
\begin{aligned}
& V^{(1)}\left(t, s_{1}, i\right)-V^{(1)}\left(t, s_{2}, i\right) \\
& \quad=\sup _{\tau \in \mathcal{T}_{t, T}} E^{\mathbb{Q}}\left[e^{-r(\tau-t)}\left(K-S_{1}(\tau)\right)^{+}\right]-\sup _{\tau \in \mathcal{T}_{t, T}} E^{\mathbb{Q}}\left[e^{-r(\tau-t)}\left(K-S_{2}(\tau)\right)^{+}\right]
\end{aligned}
$$




$$
\begin{aligned}
& \leq \sup _{\tau \in \mathcal{T}_{t, T}} E^{\mathbb{Q}}\left[e^{-r(\tau-t)}\left|\left(K-S_{1}(\tau)\right)^{+}-\left(K-S_{2}(\tau)\right)^{+}\right|\right] \\
& \leq \sup _{\tau \in \mathcal{T}_{t, T}} E^{\mathbb{Q}}\left[e^{-r(\tau-t)}\left|S_{1}(\tau)-S_{2}(\tau)\right|\right] .
\end{aligned}
$$

Here we need to obtain the upper bound of $E^{\mathbb{Q}}\left[\left|S_{1}(u)-S_{2}(u)\right|\right](u \in[t, T])$. From SDE (1), by using the Cauchy-Schwarz inequality and Tonelli's theorem we find that

$$
\begin{aligned}
E^{\mathbb{Q}}\left[\left|S_{1}(u)-S_{2}(u)\right|^{2}\right] & E^{\mathbb{Q}}\left[\left|\left(s_{1}-s_{2}\right)+\int_{t}^{u} \beta(\alpha(\tau))\left(S_{2}(\tau)-S_{1}(\tau)\right) d \tau\right|^{2}\right] \\
= & E^{\mathbb{Q}}\left[\left(s_{1}-s_{2}\right)^{2}+2\left(s_{1}-s_{2}\right) \int_{t}^{u} \beta(\alpha(\tau))\left(S_{2}(\tau)-S_{1}(\tau)\right) d \tau\right. \\
& \left.+\left(\int_{t}^{u} \beta(\alpha(\tau))\left(S_{2}(\tau)-S_{1}(\tau)\right) d \tau\right)^{2}\right] \\
\leq & 2\left|s_{1}-s_{2}\right|^{2}+2 E^{\mathbb{Q}}\left[\left(\int_{t}^{u} \beta(\alpha(\tau))\left(S_{2}(\tau)-S_{1}(\tau)\right) d \tau\right)^{2}\right] \\
\leq & 2\left|s_{1}-s_{2}\right|^{2}+C E^{\mathbb{Q}}\left[\int_{t}^{u} \beta^{2}(\alpha(\tau))\left(S_{2}(\tau)-S_{1}(\tau)\right)^{2} d \tau\right] \\
\leq & 2\left|s_{1}-s_{2}\right|^{2}+C E^{\mathbb{Q}}\left[\int_{t}^{u}\left(S_{2}(\tau)-S_{1}(\tau)\right)^{2} d \tau\right] \\
= & 2\left|s_{1}-s_{2}\right|^{2}+C \int_{t}^{u} E^{\mathbb{Q}}\left[\left(S_{2}(\tau)-S_{1}(\tau)\right)^{2}\right] d \tau .
\end{aligned}
$$

Notice that $C$ is a generic positive constant, whose value varies in different cases. Next we adopt Gronwall's inequality to deal with (10), and we obtain

$$
E^{\mathbb{Q}}\left[\left|S_{1}(u)-S_{2}(u)\right|^{2}\right] \leq C\left|s_{1}-s_{2}\right|^{2}, \quad \forall u \in[t, T]
$$

Then, by using the Cauchy-Schwarz inequality again, we get an upper bound of $E^{\mathbb{Q}}\left[\mid S_{1}(u)\right.$ $\left.S_{2}(u) \mid\right]$, that is,

$$
E^{\mathbb{Q}}\left[\left|S_{1}(u)-S_{2}(u)\right|\right] \leq C\left|s_{1}-s_{2}\right|, \quad \forall t \in[0, T]
$$

With the inequality (11), we can continue to deal with the inequality (9) and finally get

$$
V^{(1)}\left(t, s_{1}, i\right)-V^{(1)}\left(t, s_{2}, i\right) \leq C\left|s_{1}-s_{2}\right|
$$

Hence $V^{(1)}(t, s, i)$ is (Lipschitz) continuous respect to $s$.

(ii) For $n=k, k \geq 2$, we assume that

$$
V^{(k-1)}\left(t, S_{1}(t), i\right)-V^{(k-1)}\left(t, S_{2}(t), i\right) \leq C\left|s_{1}-s_{2}\right|, \quad \forall t \in[0, T],
$$

under which we can compute for $n=k$,

$$
\left|V^{(k)}\left(t, s_{1}, i\right)-V^{(k)}\left(t, s_{2}, i\right)\right| \leq \sup _{\tau \in \mathcal{T}_{t, T}} C E^{\mathbb{Q}}\left[\left|G^{(k)}\left(\tau, S_{1}(\tau), \alpha(\tau)\right)-G^{(k)}\left(\tau, S_{2}(\tau), \alpha(\tau)\right)\right|\right] .
$$


Here we need to investigate the property of $\left|G^{(k)}\left(\tau, S_{1}(\tau), \alpha(\tau)\right)-G^{(k)}\left(\tau, S_{2}(\tau), \alpha(\tau)\right)\right|$ as follows:

$$
\begin{aligned}
\mid G^{(k)} & \left(\tau, S_{1}(\tau), \alpha(\tau)\right)-G^{(k)}\left(\tau, S_{2}(\tau), \alpha(\tau)\right) \mid \\
\leq & \left|\left(K-S_{1}(\tau)\right)^{+}-\left(K-S_{2}(\tau)\right)^{+}\right| \\
& +\left|E^{\mathbb{Q}}\left[e^{-r \delta}\left(V^{(k-1)}\left(\tau+\delta, S_{1}(\tau+\delta), \alpha(\tau+\delta)\right)-V^{(k-1)}\left(\tau+\delta, S_{2}(\tau+\delta), \alpha(\tau+\delta)\right)\right)\right]\right| \\
\leq & C\left|S_{1}(\tau)-S_{2}(\tau)\right|+C E^{\mathbb{Q}}\left[\mid V^{(k-1)}\left(\tau+\delta, S_{1}(\tau+\delta), \alpha(\tau+\delta)\right)\right. \\
& \left.-V^{(k-1)}\left(\tau+\delta, S_{2}(\tau+\delta), \alpha(\tau+\delta)\right) \mid\right] \\
\leq & C\left|S_{1}(\tau)-S_{2}(\tau)\right|+C E^{\mathbb{Q}}\left[\left|S_{1}(\tau+\delta)-S_{2}(\tau+\delta)\right|\right] \\
\leq & C\left|S_{1}(\tau)-S_{2}(\tau)\right|+C\left|S_{1}-S_{2}\right| .
\end{aligned}
$$

In this way, we get

$$
\left|V^{(k)}\left(t, s_{1}, i\right)-V^{(k)}\left(t, s_{2}, i\right)\right| \leq \sup _{\tau \in \mathcal{T}_{t, T}} E^{\mathbb{Q}}\left[C\left|S_{1}(\tau)-S_{2}(\tau)\right|+C\left|s_{1}-s_{2}\right|\right] \leq C\left|s_{1}-s_{2}\right|,
$$

which completes the proof of continuity of $V^{(n)}(t, s, i)$ with respect to $s$.

Lemma 2 Let $(t, s) \in[0, T] \times \mathbb{R}^{+}$, then, for each $i \in \mathcal{M}$ and $n \in N^{+}$, the value function $V_{i}^{(n)}(t, s)$ is continuous with respect to $t$.

Proof Firstly, we define the objective function

$$
\begin{aligned}
J^{(n)}(t, s, i, \tau) & :=E^{\mathbb{Q}}\left[e^{-r(\tau-t)} G^{(n)}(\tau, S(\tau), \alpha(\tau)) \mid S(t)=s, \alpha(t)=i\right] \\
& =e^{r t} E^{\mathbb{Q}}\left[h^{(n)}(\tau, S(\tau), \alpha(\tau)) \mid S(t)=s, \alpha(t)=i\right], \quad \tau \in[t, T],
\end{aligned}
$$

where

$$
h^{(n)}(\tau, S(\tau), \alpha(\tau)):=e^{-r \tau} G^{(n)}(\tau, S(\tau), \alpha(\tau))
$$

Next, for any $t$ and $t^{\prime}$, we set $0<t<t^{\prime}<\tau<\tau^{\prime}:=\tau+t^{\prime}-t<T$, our discussion is based on mathematical induction.

(i) For $n=1$, we have

$$
\begin{aligned}
J^{(1)}(t, s, i, \tau) & :=e^{r t} E^{\mathbb{Q}}\left[h^{(1)}(\tau, S(\tau), \alpha(\tau)) \mid S(t)=s, \alpha(t)=i\right] \\
& =e^{r t^{\prime}} E^{\mathbb{Q}}\left[h^{(1)}\left(\tau^{\prime}, S\left(\tau^{\prime}\right), \alpha\left(\tau^{\prime}\right)\right) \mid S\left(t^{\prime}\right)=s, \alpha\left(t^{\prime}\right)=i\right] \\
& =e^{r t^{\prime}} E^{\mathbb{Q}}\left[h^{(1)}\left(\tau+\left(t^{\prime}-t\right), S\left(\tau^{\prime}\right), \alpha\left(\tau^{\prime}\right)\right) \mid S\left(t^{\prime}\right)=s, \alpha\left(t^{\prime}\right)=i\right],
\end{aligned}
$$

and

$$
\begin{aligned}
J^{(1)}\left(t^{\prime}, s, i, \tau^{\prime}\right) & :=e^{r t^{\prime}} E^{\mathbb{Q}}\left[h^{(1)}\left(\tau^{\prime}, S\left(\tau^{\prime}\right), \alpha\left(\tau^{\prime}\right)\right) \mid S\left(t^{\prime}\right)=s, \alpha\left(t^{\prime}\right)=i\right] \\
& =e^{r t} E^{\mathbb{Q}}\left[h^{(1)}(\tau, S(\tau), \alpha(\tau)) \mid S(t)=s, \alpha(t)=i\right]
\end{aligned}
$$




$$
=e^{r t} E^{\mathbb{Q}}\left[h^{(1)}\left(\tau^{\prime}-\left(t^{\prime}-t\right), S(\tau), \alpha(\tau)\right) \mid S(t)=s, \alpha(t)=i\right] .
$$

Then we investigate the property of $h^{(1)}(t, S(t), \alpha(t))(t \in[0, T])$. To this end, we assume that, for any $t_{1}, t_{2}>0, S\left(t_{1}\right)=s_{1}, S\left(t_{2}\right)=s_{2}$, and since $\left|e^{-x_{1}}-e^{-x_{2}}\right| \leq\left|x_{1}-x_{2}\right|$ for any $x_{1}, x_{2} \geq$ 0 , we obtain

$$
\begin{aligned}
h^{(1)} & \left(t_{1}, s_{1}, \alpha\left(t_{1}\right)\right)-h^{(1)}\left(t_{2}, s_{2}, \alpha\left(t_{2}\right)\right) \\
& \leq\left|h^{(1)}\left(t_{1}, s_{1}, \alpha\left(t_{1}\right)\right)-h^{(1)}\left(t_{2}, s_{2}, \alpha\left(t_{2}\right)\right)\right| \\
& =\left|e^{-r t_{1}}\left(K-S\left(t_{1}\right)\right)^{+}-e^{-r t_{2}}\left(K-S\left(t_{2}\right)\right)^{+}\right| \\
& \leq\left|e^{-r t_{1}}\left(K-S\left(t_{1}\right)\right)-e^{-r t_{2}}\left(K-S\left(t_{2}\right)\right)\right| \\
& =\left|K\left(e^{-r t_{1}}-e^{-r t_{2}}\right)+e^{-r t_{2}} S\left(t_{2}\right)-e^{-r t_{1}} S\left(t_{1}\right)\right| \\
& \leq K\left|e^{-r t_{1}}-e^{-r t_{2}}\right|+\left|e^{-r t_{2}} s_{2}-e^{-r t_{2}} s_{1}+e^{-r t_{2}} s_{1}-e^{-r t_{1}} s_{1}\right| \\
& \leq K\left|e^{-r t_{1}}-e^{-r t_{2}}\right|+e^{-r t_{2}}\left|s_{2}-s_{1}\right|+s_{1}\left|e^{-r t_{2}}-e^{-r t_{1}}\right| \\
& \leq K\left|t_{2}-t_{1}\right|+e^{-r t_{2}}\left|s_{2}-s_{1}\right|+s_{1}\left|t_{2}-t_{1}\right| \\
& =C\left|t_{2}-t_{1}\right|+C\left|s_{2}-s_{1}\right| .
\end{aligned}
$$

Now we proceed to dealing with the equality (12) to find

$$
\begin{aligned}
& J^{(1)}(t, s, i, \tau) \\
& \quad \leq e^{r t^{\prime}} E^{\mathbb{Q}}\left[h^{(1)}(\tau, S(\tau), \alpha(\tau))+C\left(\left|t^{\prime}-t\right|\right)+C\left|S\left(\tau^{\prime}\right)-S(\tau)\right| \mid S\left(t^{\prime}\right)=s, \alpha\left(t^{\prime}\right)=i\right] .
\end{aligned}
$$

With the fact that

$$
E^{\mathbb{Q}}\left[\left|S\left(\tau^{\prime}\right)-S(\tau)\right|\right] \leq \sqrt{E^{\mathbb{Q}}\left[\left|S\left(\tau^{\prime}\right)-S(\tau)\right|^{2}\right]} \leq C\left|\tau^{\prime}-\tau\right|^{\frac{1}{2}}=C\left|t^{\prime}-t\right|^{\frac{1}{2}},
$$

it follows from (14) that

$$
\begin{aligned}
J^{(1)} & (t, s, i, \tau) \\
& \leq e^{r t^{\prime}} E^{\mathbb{Q}}\left[h^{(1)}(\tau, S(\tau), \alpha(\tau)) \mid S\left(t^{\prime}\right)=s, \alpha\left(t^{\prime}\right)=i\right]+C\left|t^{\prime}-t\right|+C\left(\left|t^{\prime}-t\right|^{\frac{1}{2}}\right) \\
& =J^{(1)}\left(t^{\prime}, s, i, \tilde{\tau}\right)+C\left|t^{\prime}-t\right|+C\left|t^{\prime}-t\right|^{\frac{1}{2}} \\
& =J^{(1)}\left(t^{\prime}, s, i, \tilde{\tau}\right)+C\left|t^{\prime}-t\right|^{\frac{1}{2}}, \quad \tau \in[t, T], \tilde{\tau} \in\left[t^{\prime}, T\right] .
\end{aligned}
$$

Similarly, we can restart from the inequality (13) and get

$$
\begin{aligned}
J^{(1)}\left(t^{\prime}, s, i, \tau^{\prime}\right) & \leq e^{r t} E^{\mathbb{Q}}[h(\tau, S(\tau), \alpha(\tau)) \mid S(t)=s, \alpha(t)=i]+C\left|t^{\prime}-t\right|+C\left(\left|t^{\prime}-t\right|^{\frac{1}{2}}\right) \\
& =J^{(1)}(t, s, i, \tilde{\tau})+C\left|t^{\prime}-t\right|+C\left|t^{\prime}-t\right|^{\frac{1}{2}} \\
& =J^{(1)}(t, s, i, \tilde{\tau})+C\left|t^{\prime}-t\right|^{\frac{1}{2}}, \quad \tau^{\prime} \in\left[t^{\prime}, T\right], \tilde{\tau} \in[t, T] .
\end{aligned}
$$


Finally, we get

$$
\begin{aligned}
& \left|V^{(1)}\left(t^{\prime}, s, i\right)-V^{(1)}(t, s, i)\right| \\
& \quad \leq \sup _{\hat{\tau} \in \mathcal{T}_{t^{\prime}, T}, \tau \in \mathcal{T}_{t, T}}\left|J^{(1)}\left(t^{\prime}, s, i, \hat{\tau}\right)-J^{(1)}(t, s, i, \tau)\right| \\
& \quad \leq C\left|t^{\prime}-t\right|^{\frac{1}{2}}, \quad 0<t<t^{\prime}<T
\end{aligned}
$$

and this indicates the continuity of $V^{(1)}$ with respect to $t$.

(ii) For $n=k, k \geq 2$, we assume that

$$
\left|V^{(k-1)}\left(t^{\prime}, s, i\right)-V^{(k-1)}(t, s, i)\right| \leq C\left|t^{\prime}-t\right|^{\frac{1}{2}}, \quad 0<t<t^{\prime}<T .
$$

Then we start from defining

$$
\begin{aligned}
J^{(k)}(t, s, i, \tau) & :=e^{r t} E^{\mathbb{Q}}\left[h^{(k)}(\tau, S(\tau), \alpha(\tau)) \mid S(t)=s, \alpha(t)=i\right] \\
& =e^{r t^{\prime}} E^{\mathbb{Q}}\left[h^{(k)}\left(\tau^{\prime}, S\left(\tau^{\prime}\right), \alpha\left(\tau^{\prime}\right)\right) \mid S\left(t^{\prime}\right)=s, \alpha\left(t^{\prime}\right)=i\right] \\
& =e^{r t^{\prime}} E^{\mathbb{Q}}\left[h^{(k)}\left(\tau+\left(t^{\prime}-t\right), S\left(\tau^{\prime}\right), \alpha\left(\tau^{\prime}\right)\right) \mid S\left(t^{\prime}\right)=s, \alpha\left(t^{\prime}\right)=i\right]
\end{aligned}
$$

and

$$
\begin{aligned}
J^{(k)}\left(t^{\prime}, s, i, \tau^{\prime}\right) & :=e^{r t^{\prime}} E^{\mathbb{Q}}\left[h^{(k)}\left(\tau^{\prime}, S\left(\tau^{\prime}\right), \alpha\left(\tau^{\prime}\right)\right) \mid S\left(t^{\prime}\right)=s, \alpha\left(t^{\prime}\right)=i\right] \\
& =e^{r t} E^{\mathbb{Q}}\left[h^{(k)}(\tau, S(\tau), \alpha(\tau)) \mid S(t)=s, \alpha(t)=i\right] \\
& =e^{r t} E^{\mathbb{Q}}\left[h^{(k)}\left(\tau^{\prime}-\left(t^{\prime}-t\right), S(\tau), \alpha(\tau)\right) \mid S(t)=s, \alpha(t)=i\right] .
\end{aligned}
$$

Next we need to investigate the property of $h^{(k)}(t, S(t), \alpha(t))(t \in[0, T])$. For this purpose, let $t_{1}, t_{2}>0, S\left(t_{1}\right)=s_{1}, S\left(t_{2}\right)=s_{2}$, and we estimate

$$
\begin{aligned}
h^{(k)} & \left(t_{1}, s_{1}, \alpha\left(t_{1}\right)\right)-h^{(k)}\left(t_{2}, s_{2}, \alpha\left(t_{2}\right)\right) \\
\leq & \left|h^{(k)}\left(t_{1}, s_{1}, \alpha\left(t_{1}\right)\right)-h^{(k)}\left(t_{2}, s_{2}, \alpha\left(t_{2}\right)\right)\right| \\
= & \left|e^{-r t_{1}} G^{(k)}\left(t_{1}, S\left(t_{1}\right), \alpha\left(t_{1}\right)\right)-e^{-r t_{2}} G^{(k)}\left(t_{2}, S\left(t_{2}\right), \alpha\left(t_{2}\right)\right)\right| \\
\leq & \left|e^{-r t_{1}} G^{(k)}\left(t_{1}, S\left(t_{1}\right), \alpha\left(t_{1}\right)\right)-e^{-r t_{1}} G^{(k)}\left(t_{2}, S\left(t_{2}\right), \alpha\left(t_{2}\right)\right)\right| \\
& \quad+\left|e^{-r t_{1}} G^{(k)}\left(t_{2}, S\left(t_{2}\right), \alpha\left(t_{1}\right)\right)-e^{-r t_{2}} G^{(k)}\left(t_{2}, S\left(t_{2}\right), \alpha\left(t_{2}\right)\right)\right| \\
:= & I_{1}+I_{2},
\end{aligned}
$$

here we need to deal with $I_{1}$ and $I_{2}$. Note that

$$
\begin{aligned}
I_{1} & =\left|e^{-r t_{1}} G^{(k)}\left(t_{1}, S\left(t_{1}\right), \alpha\left(t_{1}\right)\right)-e^{-r t_{1}} G^{(k)}\left(t_{2}, S\left(t_{2}\right), \alpha\left(t_{2}\right)\right)\right| \\
& \leq e^{-r t_{1}}\left|G^{(k)}\left(t_{1}, S\left(t_{1}\right), \alpha\left(t_{1}\right)\right)-G^{(k)}\left(t_{2}, S\left(t_{2}\right), \alpha\left(t_{2}\right)\right)\right| \\
& =C\left|G^{(k)}\left(t_{1}, S\left(t_{1}\right), \alpha\left(t_{1}\right)\right)-G^{(k)}\left(t_{2}, S\left(t_{2}\right), \alpha\left(t_{2}\right)\right)\right| \\
& \leq C\left(\left|G^{(k)}\left(t_{1}, S\left(t_{1}\right), \alpha\left(t_{1}\right)\right)-G^{(k)}\left(t_{1}, S\left(t_{2}\right), \alpha\left(t_{2}\right)\right)\right|\right.
\end{aligned}
$$




$$
\begin{aligned}
& \left.+\left|G^{(k)}\left(t_{1}, S\left(t_{2}\right), \alpha\left(t_{1}\right)\right)-G^{(k)}\left(t_{2}, S\left(t_{2}\right), \alpha\left(t_{2}\right)\right)\right|\right) \\
:= & C\left(I_{11}+I_{12}\right),
\end{aligned}
$$

where

$$
\begin{aligned}
I_{11}= & \left|G^{(k)}\left(t_{1}, S\left(t_{1}\right), \alpha\left(t_{1}\right)\right)-G^{(k)}\left(t_{1}, S\left(t_{2}\right), \alpha\left(t_{2}\right)\right)\right| \\
= & \left|G^{(k)}\left(t_{1}, s_{1}, \alpha\left(t_{1}\right)\right)-G^{(k)}\left(t_{1}, s_{2}, \alpha\left(t_{1}\right)\right)\right| \\
= & \mid\left(K-s_{1}\right)^{+}+E^{\mathbb{Q}}\left[e^{-r \delta} V^{(k-1)}\left(t_{1}+\delta, S_{1}\left(t_{1}+\delta\right), \alpha\left(t_{1}+\delta\right)\right)\right] \\
& -\left(K-s_{2}\right)^{+}-E^{\mathbb{Q}}\left[e^{-r \delta} V^{(k-1)}\left(t_{1}+\delta, S_{2}\left(t_{1}+\delta\right), \alpha\left(t_{1}+\delta\right)\right)\right] \mid \\
\leq & \left|s_{2}-s_{1}\right|+C E^{\mathbb{Q}}\left[\mid V^{(k-1)}\left(t_{1}+\delta, S_{1}\left(t_{1}+\delta\right), \alpha\left(t_{1}+\delta\right)\right)\right. \\
& \left.-V^{(k-1)}\left(t_{1}+\delta, S_{2}\left(t_{1}+\delta\right), \alpha\left(t_{1}+\delta\right)\right) \mid\right] \\
\leq & \left|s_{2}-s_{1}\right|+C E^{\mathbb{Q}}\left[\left|S_{2}\left(t_{1}+\delta\right)-S_{1}\left(t_{1}+\delta\right)\right|\right] \\
\leq & C\left|s_{2}-s_{1}\right|
\end{aligned}
$$

and

$$
\begin{aligned}
I_{12}= & \left|G^{(k)}\left(t_{1}, S\left(t_{2}\right), \alpha\left(t_{1}\right)\right)-G^{(k)}\left(t_{2}, S\left(t_{2}\right), \alpha\left(t_{2}\right)\right)\right| \\
= & \mid E^{\mathbb{Q}}\left[e^{-r \delta} V^{(k-1)}\left(t_{1}+\delta, S\left(t_{1}+\delta\right), \alpha\left(t_{1}+\delta\right)\right) \mid S\left(t_{1}\right)=s_{2}, \alpha\left(t_{1}\right)=i\right] \\
& -E^{\mathbb{Q}}\left[e^{-r \delta} V^{(k-1)}\left(t_{2}+\delta, S\left(t_{2}+\delta\right), \alpha\left(t_{2}+\delta\right)\right) \mid S\left(t_{2}\right)=s_{2}, \alpha\left(t_{2}\right)=i\right] \mid \\
= & \mid E^{\mathbb{Q}}\left[e^{-r \delta} V^{(k-1)}\left(t_{1}+\delta, S^{t_{1}, s_{2}, i}\left(t_{1}+\delta\right), \alpha\left(t_{1}+\delta\right)\right)\right] \\
& -E^{\mathbb{Q}}\left[e^{-r \delta} V^{(k-1)}\left(t_{2}+\delta, S^{t_{2}, s_{2}, i}\left(t_{2}+\delta\right), \alpha\left(t_{2}+\delta\right)\right)\right] \mid \\
\leq & E^{\mathbb{Q}}\left[C \mid V^{(k-1)}\left(t_{1}+\delta, S^{t_{1}, s_{2}, i}\left(t_{1}+\delta\right), \alpha\left(t_{1}+\delta\right)\right)\right. \\
& \left.-V^{(k-1)}\left(t_{2}+\delta, S^{t_{2}, s_{2}, i}\left(t_{2}+\delta\right), \alpha\left(t_{2}+\delta\right)\right) \mid\right] \\
\leq & E^{\mathbb{Q}}\left[C\left|t_{2}-t_{1}\right|^{\frac{1}{2}}\right] \quad\left(\text { since } S^{t_{1}, s_{2}, i}\left(t_{1}+\delta\right)=S^{t_{2}, s_{2}, i}\left(t_{2}+\delta\right)\right) \\
\leq & C\left|t_{2}-t_{1}\right|^{\frac{1}{2}},
\end{aligned}
$$

and in this way we get

$$
\begin{aligned}
I_{1} & =\left|e^{-r t_{1}} G^{(k)}\left(t_{1}, S\left(t_{1}\right), \alpha\left(t_{1}\right)\right)-e^{-r t_{1}} G^{(k)}\left(t_{2}, S\left(t_{2}\right), \alpha\left(t_{2}\right)\right)\right| \\
& \leq C\left|s_{2}-s_{1}\right|+C\left|t_{2}-t_{1}\right|^{\frac{1}{2}} .
\end{aligned}
$$

For $I_{2}$, we get

$$
\begin{aligned}
I_{2} & =\left|e^{-r t_{1}} G^{(k)}\left(t_{2}, S\left(t_{2}\right), \alpha\left(t_{2}\right)\right)-e^{-r t_{2}} G^{(k)}\left(t_{2}, S\left(t_{2}\right), \alpha\left(t_{2}\right)\right)\right| \\
& \leq G^{(k)}\left(t_{2}, S\left(t_{2}\right), \alpha\left(t_{2}\right)\right)\left|e^{-r t_{1}}-e^{-r t_{2}}\right| \\
& \leq C\left|e^{-r t_{1}}-e^{-r t_{2}}\right| \\
& \leq C\left|t_{2}-t_{1}\right| .
\end{aligned}
$$


Hence we can conclude that

$$
\left|h^{(k)}\left(t_{1}, s_{1}, \alpha\left(t_{1}\right)\right)-h^{(k)}\left(t_{2}, s_{2}, \alpha\left(t_{2}\right)\right)\right| \leq C\left|s_{2}-s_{1}\right|+C\left|t_{2}-t_{1}\right|^{\frac{1}{2}}+C\left|t_{2}-t_{1}\right| .
$$

Now we come back to Eq. (15), and we estimate

$$
\begin{aligned}
& J^{(k)}(t, s, i, \tau) \\
& \quad \leq e^{r t^{\prime}} E^{\mathbb{Q}}\left[h^{(k)}(\tau, S(\tau))+C\left|S\left(\tau^{\prime}\right)-S(\tau)\right|+C\left|t^{\prime}-t^{\frac{1}{2}}+C\right| t^{\prime}-t|| S\left(t^{\prime}\right)=s, \alpha\left(t^{\prime}\right)=i\right] .
\end{aligned}
$$

With the fact that

$$
E^{\mathbb{Q}}\left[\left|S\left(\tau^{\prime}\right)-S(\tau)\right|\right] \leq \sqrt{E^{\mathbb{Q}}\left[\left|S\left(\tau^{\prime}\right)-S(\tau)\right|^{2}\right]} \leq C\left|\tau^{\prime}-\tau\right|^{\frac{1}{2}}=C\left|t^{\prime}-t\right|^{\frac{1}{2}}
$$

we find that

$$
\begin{aligned}
J^{(k)}(t, s, i, \tau) & \leq e^{r t^{\prime}} E^{\mathbb{Q}}\left[h^{(k)}(\tau, S(\tau), \alpha(\tau)) \mid S\left(t^{\prime}\right)=s, \alpha\left(t^{\prime}\right)=i\right]+C\left|t^{\prime}-t\right|+C\left(\left|t^{\prime}-t\right|^{\frac{1}{2}}\right) \\
& =J^{(k)}\left(t^{\prime}, s, i, \tilde{\tau}\right)+C\left|t^{\prime}-t\right|+C\left|t^{\prime}-t\right|^{\frac{1}{2}} \\
& =J^{(k)}\left(t^{\prime}, s, i, \tilde{\tau}\right)+C\left|t^{\prime}-t\right|^{\frac{1}{2}}, \quad \tau \in[t, T], \tilde{\tau} \in\left[t^{\prime}, T\right] .
\end{aligned}
$$

Similarly, we can restart from the equality (16) to get

$$
\begin{aligned}
J^{(k)}\left(t^{\prime}, s, i, \tau^{\prime}\right) & \leq e^{r t} E^{\mathbb{Q}}[h(\tau, S(\tau), \alpha(\tau)) \mid S(t)=s, \alpha(t)=i]+C\left|t^{\prime}-t\right|+C\left(\left|t^{\prime}-t\right|^{\frac{1}{2}}\right) \\
& =J^{(k)}(t, s, i, \tilde{\tau})+C\left|t^{\prime}-t\right|+C\left|t^{\prime}-t\right|^{\frac{1}{2}} \\
& =J^{(k)}(t, s, i, \tilde{\tau})+C\left|t^{\prime}-t\right|^{\frac{1}{2}}, \quad \tau^{\prime} \in\left[t^{\prime}, T\right], \tilde{\tau} \in[t, T] .
\end{aligned}
$$

In this way, we finally arrive at the estimation that

$$
\begin{aligned}
\left|V^{(k)}\left(t^{\prime}, s, i\right)-V^{(k)}(t, s, i)\right| & \leq \sup _{\hat{\tau} \in \mathcal{T}_{t^{\prime}, T}, \tau \in \mathcal{T}_{t, T}}\left|J^{(k)}\left(t^{\prime}, s, i, \hat{\tau}\right)-J^{(k)}(t, s, i, \tau)\right| \\
& \leq C\left|t^{\prime}-t\right|^{\frac{1}{2}}
\end{aligned}
$$

and this indicates the continuity of $V^{(k)}$ with respect to $t$. Therefore we complete the proof of the continuity of $V^{(n)}$ with respect to $t$.

Lemma 3 Let $(t, s) \in[0, T] \times \mathbb{R}^{+}$, then, for each $i \in \mathcal{M}$ and $n \in N^{+}$, there exists a constant C such that $\left|V^{(n)}(t, s, i)\right| \leq C(1+|s|)$.

Proof For each $i \in \mathcal{M}$, given any $t \in[0, T]$, assume that $S(t)=s$ and $\alpha(t)=i$. Then, by mathematical induction, we have the following.

(i) For $n=1$,

$$
\left|V^{(1)}(t, s, i)\right|=\sup _{\tau \in \mathcal{T}_{t, T}} E^{\mathbb{Q}}\left[e^{-r(\tau-t)} G_{j}^{(1)}(\tau, S(\tau)) \mid S(t)=s, \alpha(t)=i\right]
$$




$$
\begin{aligned}
& =\sup _{\tau \in \mathcal{T}_{t, T}} E^{\mathbb{Q}}\left[e^{-r(\tau-t)}(K-S(\tau))^{+} \mid S(t)=s, \alpha(t)=i\right] \\
& \leq \sup _{\tau \in \mathcal{T}_{t, T}} E^{\mathbb{Q}}[C|K-S(\tau)| \mid S(t)=s, \alpha(t)=i] \\
& \leq \sup _{\tau \in \mathcal{T}_{t, T}} E^{\mathbb{Q}}[C(|K-S(t)|+|S(t)-S(\tau)|) \mid S(t)=s, \alpha(t)=i] \\
& \leq \sup _{\tau \in \mathcal{T}_{t, T}} E^{\mathbb{Q}}[C(K+|s|+|S(t)-S(\tau)|) \mid S(t)=s, \alpha(t)=i] \\
& =\sup _{\tau \in \mathcal{T}_{t, T}} C(K+|s|)+E^{\mathbb{Q}}[C|S(t)-S(\tau)| \mid S(t)=s, \alpha(t)=i] \\
& \leq \sup _{\tau \in \mathcal{T}_{t, T}} C(K+|s|)+C|\tau-t|^{\frac{1}{2}} \leq C(K+|s|)+C T^{\frac{1}{2}}=C(1+|s|) .
\end{aligned}
$$

(ii) For $n=k, k \geq 2$, assuming that

$$
\left|V^{(k-1)}(t, s, i)\right| \leq C(1+|s|), \quad \forall t \in[0, T]
$$

then using the results in Lemma 1 and Lemma 2, we get

$$
\begin{aligned}
\left|V^{(k)}(t, s, i)\right|= & \sup _{\tau \in \mathcal{T}_{t, T}} E^{\mathbb{Q}}\left[e^{-r(\tau-t)} G^{(k)}(\tau, S(\tau), \alpha(\tau)) \mid S(t)=s, \alpha(t)=i\right] \\
\leq & \sup _{\tau \in \mathcal{T}_{t, T}}\left(C E^{\mathbb{Q}}\left[(K-S(\tau))^{+} \mid S(t)=s, \alpha(t)=i\right]\right. \\
& \left.+C E^{\mathbb{Q}}\left[\left|V^{(k-1)}(\tau+\delta, S(\tau+\delta), j)\right| \mid S(t)=s, \alpha(t)=i\right]\right) \\
\leq & C(1+|s|)+\sup _{\tau \in \mathcal{T}_{t, T}} E^{\mathbb{Q}}\left[C \left(\left|V^{(k-1)}(t, s, j)\right|+C|\tau+\delta-t|^{\frac{1}{2}}\right.\right. \\
& +C|S(\tau+\delta)-S(t)|) \mid S(t)=s, \alpha(t)=i] \\
\leq & C(1+|s|)+\sup _{\tau \in \mathcal{T}_{t, T}} E^{\mathbb{Q}}\left[C\left(C(1+|s|)+|\tau+\delta-t|^{\frac{1}{2}}+C|\tau+\delta-t|^{\frac{1}{2}}\right)\right. \\
\leq & C(1+|s|) .
\end{aligned}
$$

\section{Existence of the viscosity solution}

This section shows the existence result. We break the whole proof into two parts, where the first part corresponds to the supersolution property of the value function, and in the second part we deal with the subsolution property.

Lemma 4 For any $n \in \mathbb{N}^{+},\left(V^{(n)}(t, s, 1), V^{(n)}(t, s, 2)\right)$ is the viscosity supersolution of $(7)$.

Proof We only need to show that, for any $i \in \mathcal{M}, V^{(n)}(t, s, i)$ is a viscosity supersolution to

$$
\left\{\begin{array}{l}
\min \left\{-\mathcal{L}_{i} W-q_{i j}\left(V_{j}^{(n)}-W\right), W-G_{i}^{(n)}\right\}=0, \quad s>0,0<t<T \\
W=(K-s)^{+}, \quad s>0, t=T
\end{array}\right.
$$

where $\mathcal{L}_{i} V$ and $G_{i}^{(n)}$ are the same as in Propersition 1. 
Given $(\bar{t}, \bar{s}) \in[0, T) \times \mathbb{R}^{+}$, assume that $\alpha(\bar{t})=i \in \mathcal{M}$, and let $\phi \in C^{1,2}\left([0, T) \times \mathbb{R}^{+}\right)$such that

$$
0=V^{(n)}(\bar{t}, \bar{s}, i)-\phi(\bar{t}, \bar{s})=\text { strict } \min _{(t, s) \in(0, T] \times \mathbb{R}^{+}}\left(V^{(n)}(t, s, i)-\phi(t, s)\right) .
$$

Let $\left\{\left(t_{m}, s_{m}\right)\right\}$ be a sequence in $[0, T] \times \mathbb{R}^{+}$such that

$$
\left\{\left(t_{m}, s_{m}\right)\right\} \rightarrow(\bar{t}, \bar{s}) \quad \text { and } \quad V_{i}^{(n)}\left(t_{m}, s_{m}\right) \rightarrow V_{i}^{(n)}(\bar{t}, \bar{s})
$$

Since $\phi \in C^{1,2}$, we have

$$
\eta_{m}:=V^{(n)}\left(t_{m}, s_{m}, i\right)-\phi\left(t_{m}, s_{m}\right) \rightarrow 0
$$

Next, let $\theta$ be the time such that $\alpha(t) \equiv i$ in $[\bar{t}, \theta]$. In addition, we denote by $S^{m}(t):=$ $S^{t_{m}, s_{m}, i}(t)$ the associated state process with initial data $S^{m}\left(t_{m}\right)=s_{m}$. For $m$ sufficiently large, we have

$$
0<h_{m}:=\eta_{m}^{\frac{1}{2}} \mathbf{1}_{\left\{\eta_{m} \neq 0\right\}}+\frac{1}{m} \mathbf{1}_{\left\{\eta_{m}=0\right\}} \leq \theta-t_{m}
$$

Then we define the stopping time

$$
\gamma_{m}:=\inf \left\{t>t_{m}:\left(t-t_{m}, S^{m}(t)-s_{m}\right) \notin\left[0, h_{m}\right) \times[-\alpha, \alpha]\right\},
$$

where $\alpha>0$ is some given constant. By the dynamic programming principle we have

$$
V^{(n)}\left(t_{m}, s_{m}, i\right) \geq E^{\mathbb{Q}}\left[e^{-r\left(\gamma_{m}-t_{m}\right)} V\left(\gamma_{m}, S^{m}\left(\gamma_{m}\right), i\right)\right]
$$

and, together with (18) and (19), we have

$$
0 \leq E^{\mathbb{Q}}\left[\eta_{m}+\phi\left(t_{m}, s_{m}\right)-e^{-r\left(\gamma_{m}-t_{m}\right)} \phi\left(\gamma_{m}, S^{m}\left(\gamma_{m}\right)\right)\right]
$$

Similar to [20], we set

$$
\psi(t, S(t), \alpha(t))= \begin{cases}\phi(t, S(t)), & \text { if } \alpha(t)=i \\ V^{(n)}(t, S(t), \alpha(t)), & \text { otherwise }\end{cases}
$$

Therefore by Dynkin's formula, we continue the inequality (20) as follows:

$$
\begin{aligned}
0 \leq & \eta_{m}+E^{\mathbb{Q}}\left[\psi\left(t_{m}, s_{m}, i\right)-e^{-r\left(\gamma_{m}-t_{m}\right)} \psi\left(\gamma_{m}, S^{m}\left(\gamma_{m}\right), i\right)\right] \\
= & \eta_{m}-E^{\mathbb{Q}}\left[\int_{t_{m}}^{\gamma_{m}} d\left(e^{-r\left(\tau-t_{m}\right)} \psi\left(\tau, S^{m}(\tau), i\right)\right)\right] \\
= & \eta_{m}-E^{\mathbb{Q}}\left[\int _ { t _ { m } } ^ { \gamma _ { m } } e ^ { - r ( \tau - t _ { m } ) } \left(\frac{\partial \psi\left(\tau, S^{m}(\tau), i\right)}{\partial t}+\frac{1}{2} \sigma^{2}(i) \frac{\partial^{2} \psi\left(\tau, S^{m}(\tau), i\right)}{\partial s^{2}}\right.\right. \\
& +\beta(i)\left(\xi(i)-S^{m}(\tau)\right) \frac{\partial \psi\left(\tau, S^{m}(\tau), i\right)}{\partial s}
\end{aligned}
$$




$$
\begin{aligned}
& \left.\left.-r \psi\left(\tau, S^{m}(\tau), i\right)+q_{i j}\left(\psi\left(\tau, S^{m}(\tau), j\right)-\psi\left(\tau, S^{m}(\tau), i\right)\right)\right) d t\right] \\
& +E^{\mathbb{Q}}\left[\int_{t_{m}}^{\gamma_{m}} e^{-r\left(\tau-t_{m}\right)} d M(\tau)\right] \\
= & \eta_{m}-E^{\mathbb{Q}}\left[\int _ { t _ { m } } ^ { \gamma _ { m } } e ^ { - r ( \tau - t _ { m } ) } \left(\frac{\partial \psi\left(\tau, S^{m}(\tau), i\right)}{\partial t}+\frac{1}{2} \sigma^{2}(i) \frac{\partial^{2} \psi\left(\tau, S^{m}(\tau), i\right)}{\partial s^{2}}\right.\right. \\
& +\beta(i)\left(\xi(i)-S^{m}(\tau)\right) \frac{\partial \psi\left(\tau, S^{m}(\tau), i\right)}{\partial s} \\
& \left.\left.-r \psi\left(\tau, S^{m}(\tau), i\right)+q_{i j}\left(V^{(n)}\left(\tau, S^{m}(\tau), j\right)-\psi\left(\tau, S^{m}(\tau), i\right)\right)\right) d t\right], \quad j \neq i .
\end{aligned}
$$

Next we divide both sides by $\gamma_{m}-t_{m}$, then the mean value theorem leads us to

$$
\begin{aligned}
& \frac{\eta_{m}}{\gamma_{m}-t_{m}}-e^{-r\left(\tau-t_{m}\right)}\left(\frac{\partial \psi\left(\tau, S^{m}(\tau), i\right)}{\partial t}+\frac{1}{2} \sigma^{2}(i) \frac{\partial^{2} \psi\left(\tau, S^{m}(\tau), i\right)}{\partial s^{2}}\right. \\
& \quad+\beta(i)\left(\xi(i)-S^{m}(\tau)\right) \frac{\partial \psi\left(\tau, S^{m}(\tau), i\right)}{\partial s} \\
& \quad-r \psi\left(\tau, S^{m}(\tau), i\right) \\
& \quad+q_{i j}\left(V^{(n)}\left(\tau, S^{m}(\tau), j\right)-\psi\left(\tau, S^{m}(\tau), i\right)\right) \geq 0, \quad \tau \in\left[t_{m}, \gamma_{m}\right], j \neq i,
\end{aligned}
$$

Note that, for $m$ sufficiently large, $\gamma_{m}=t_{m}+h_{m}$, therefore

$$
\frac{\eta_{m}}{\gamma_{m}-t_{m}}=\frac{\eta_{m}}{h_{m}}= \begin{cases}0 \cdot \frac{1}{m}, & \text { if } \eta_{m}=0 \\ \sqrt{\eta_{m}}, & \text { otherwise. }\end{cases}
$$

Finally, we send $m$ to infinity, and the inequality (21) becomes

$$
\begin{aligned}
& -\frac{\partial \phi(\bar{t}, \bar{s})}{\partial t}-\frac{1}{2} \sigma^{2}(i) \frac{\partial^{2} \phi(\bar{t}, \bar{s})}{\partial s^{2}}-\beta(i)(\xi(i)-\bar{s}) \frac{\partial \phi(\bar{t}, \bar{s})}{\partial s}+r \phi(\bar{t}, \bar{s})-q_{i j}\left(V^{(n)}(\bar{t}, \bar{s}, j)-\phi(\bar{t}, \bar{s})\right) \\
& \quad \geq 0, \quad j \neq i .
\end{aligned}
$$

In addition, Theorem 1 states

$$
V^{(n)}(\bar{t}, \bar{s}, i)-G^{(n)}(\bar{t}, \bar{s}, i) \geq 0
$$

and together with Lemma 1 , we find that $V^{(n)}(t, s, i)$ is the continuous viscosity supersolution of (17).

Lemma 5 For any $n \in \mathbb{N}^{+},\left(V^{(n)}(t, s, 1), V^{(n)}(t, s, 2)\right)$ is the viscosity subsolution of $(7)$.

Proof We only need to show that, for any $i \in \mathcal{M}, V^{(n)}(t, s, i)$ is a viscosity subsolution to

$$
\left\{\begin{array}{l}
\min \left\{-\mathcal{L}_{i} W-q_{i j}\left(V_{j}^{(n)}-W\right), W-G_{i}^{(n)}\right\}=0, \quad s>0,0<t<T, \\
W=(K-s)^{+}, \quad s>0, t=T
\end{array}\right.
$$

where $\mathcal{L}_{i} V$ and $G_{i}^{(n)}$ are the same as in Lemma 1. 
We argue by contradiction. Given $(\bar{t}, \bar{s}) \in[0, T) \times \mathbb{R}^{+}$, assume $\alpha(\bar{t})=i \in \mathcal{M}$ and let $\phi^{1,2}\left([0, T) \times \mathbb{R}^{+}\right)$such that

$$
0=V^{(n)}(\bar{t}, \bar{s}, i)-\phi(\bar{t}, \bar{s})=\text { strict } \max _{(t, s) \in(0, T] \times \mathbb{R}^{+}}\left(V^{(n)}(t, s, i)-\phi(t, s)\right) .
$$

We assume to the contrary that

$$
\begin{aligned}
& -\frac{\partial \phi(\bar{t}, \bar{s})}{\partial t}-\frac{1}{2} \sigma^{2}(i) \frac{\partial^{2} \phi(\bar{t}, \bar{s})}{\partial s^{2}}-\beta(i)(\xi(i)-\bar{s}) \frac{\partial \phi(\bar{t}, \bar{s})}{\partial s}+r \phi(\bar{t}, \bar{s})-q_{i j}\left(V^{(n)}(\bar{t}, \bar{s}, j)-\phi(\bar{t}, \bar{s})\right) \\
& >0, \quad j \neq i,
\end{aligned}
$$

and

$$
V^{(n)}(\bar{t}, \bar{s}, i)-g(\bar{t}, \bar{s}, i)>0
$$

Next, let $\theta$ be the time such that $\alpha(t) \equiv i$ in $[\bar{t}, \theta]$. We try to work towards a contradiction of the weak dynamic programming principle, i.e.,

$$
\begin{aligned}
& V^{(n)}(\bar{t}, \bar{s}, i) \\
& \quad \leq E^{\mathbb{Q}}\left[e ^ { - r ( \tau \wedge \tau ^ { \prime } - \overline { t } ) } \left(\mathbf{1}_{\left\{\tau<\tau^{\prime}\right\}} G^{(n)}(\tau, S(\tau), i)\right.\right. \\
& \left.\left.\quad+\mathbf{1}_{\left\{\tau \geq \tau^{\prime}\right\}} V^{(n)}\left(\tau^{\prime}, S\left(\tau^{\prime}\right), i\right)\right) \mid S(\bar{t})=\bar{s}, \alpha(\bar{t})=i\right]
\end{aligned}
$$

for all $(\bar{t}, \bar{s}) \in[0, T] \times \mathbb{R}^{+}$and $\tau^{\prime} \in \mathcal{T}_{\bar{t}, \theta}$ such that $S^{\bar{t}, \bar{s}}\left(\tau^{\prime}\right)$ is $L^{\infty}$-bounded.

Firstly by the properties that $G^{(n)} \in C\left([0, T] \times \mathbb{R}^{+} \times \mathcal{M}\right)$ and the first equality of (23), we know that there exist $\delta>0$ and $0<h \leq \theta$ such that the following inequalities hold:

$$
\begin{gathered}
-\frac{\partial \phi(\bar{t}, \bar{s})}{\partial t}-\frac{1}{2} \sigma^{2}(i) \frac{\partial^{2} \phi(\bar{t}, \bar{s})}{\partial s^{2}}-\beta(i)(\xi(i)-\bar{s}) \frac{\partial \phi(\bar{t}, \bar{s})}{\partial s} \\
+r \phi(\bar{t}, \bar{s})-q_{i j}\left(V^{(n)}(\bar{t}, \bar{s}, j)-\phi(\bar{t}, \bar{s})\right) \geq 0, \quad j \neq i
\end{gathered}
$$

and

$$
\phi(t, s)-G^{(n)}(t, s, i) \geq V^{(n)}(t, s, i)-G^{(n)}(t, s, i) \geq \delta
$$

on $D:=[\bar{t}, \bar{t}+h] \times[\bar{s}-\delta, \bar{s}+\delta]$. Secondly, by the second equality of (23), we get

$$
-\gamma:=\max _{\partial D}\left(V^{(n)}(\cdot, \cdot, i)-\phi(\cdot, \cdot, i)\right)<0 .
$$

Thirdly, by the continuity of $V^{(n)}(t, s, i)$, there exists a sequence $\left\{\left(t_{m}, s_{m}\right)\right\}$ in $(0, T] \times \mathbb{R}^{+}$ such that

$$
\left(t_{m}, s_{m}\right) \rightarrow(\bar{t}, \bar{s}) \quad \text { and } \quad V\left(t_{m}, s_{m}, i\right) \rightarrow V(\bar{t}, \bar{s}, i) \quad \text { as } m \rightarrow \infty
$$

Next we define the stopping time,

$$
\gamma_{m}:=\inf \left\{t>t_{m} \mid\left(t, S^{t_{m}, s_{m}}(t)\right) \notin D\right\}
$$


and denote

$$
\eta_{m}:=V^{(n)}\left(t_{m}, s_{m}, i\right)-\phi\left(t_{m}, s_{m}\right) \rightarrow 0 .
$$

Then, for arbitrary stopping time $\tau \in \mathcal{T}_{t_{m}, T}$,

$$
V^{(n)}\left(t_{m}, s_{m}, i\right)=\eta_{m}+\phi\left(t_{m}, s_{m}\right)
$$

And we define

$$
\psi(t, S(t), \alpha(t))= \begin{cases}\phi(t, S(t)), & \text { if } \alpha(t)=i \\ V^{(n)}(t, S(t), \alpha(t)), & \text { otherwise }\end{cases}
$$

Therefore,

$$
\begin{aligned}
V^{(n)}( & \left.t_{m}, s_{m}, i\right) \\
= & \eta_{m}+\phi\left(t_{m}, s_{m}\right) \\
= & \eta_{m}+E^{\mathbb{Q}}\left[e^{-r\left(\tau \wedge \gamma_{m}-t_{m}\right)} \psi\left(\tau \wedge \gamma_{m}, S\left(\tau \wedge \gamma_{m}\right), i\right)-\int_{t_{m}}^{\tau \wedge \gamma_{m}} e^{-r\left(t-t_{m}\right)}\left(\frac{\partial \psi(t, S(t), i)}{\partial t}\right.\right. \\
& +\frac{1}{2} \sigma^{2}(i) \frac{\partial^{2} \phi(t, S(t), i)}{\partial s^{2}}+\beta(i)(\xi(i)-S(t)) \frac{\partial \psi(t, S(t), i)}{\partial s}-r \psi(t, S(t), i) \\
& \left.+q_{i j}(\psi(t, S(t), j)-\psi(t, S(t), i))\right) d t \\
& \left.+\int_{t_{m}}^{\tau \wedge \gamma_{m}} e^{-r\left(t-t_{m}\right)} d M(t) \mid S\left(t_{m}\right)=s_{m}, \alpha\left(t_{m}\right)=i\right], \quad j \in \mathcal{M}, j \neq \alpha(t) \\
= & \eta_{m}+E^{\mathbb{Q}}\left[e^{-r\left(\tau \wedge \gamma_{m}-t_{m}\right)} \psi\left(\tau \wedge \gamma_{m}, S\left(\tau \wedge \gamma_{m}\right), i\right)-\int_{t_{m}}^{\tau \wedge \gamma_{m}} e^{-r\left(t-t_{m}\right)}\left(\frac{\partial \psi(t, S(t), i)}{\partial t}\right.\right. \\
& +\frac{1}{2} \sigma^{2}(i) \frac{\partial^{2} \phi(t, S(t), i)}{\partial s^{2}}+\beta(i)(\xi(i)-S(t)) \frac{\partial \psi(t, S(t), i)}{\partial s}-r \psi(t, S(t), i) \\
& \left.\left.+q_{i j}\left(V^{(n)}(t, S(t), j)-\psi(t, S(t), i)\right)\right) d t \mid S\left(t_{m}\right)=s_{m}, \alpha\left(t_{m}\right)=i\right], \quad j \in \mathcal{M}, j \neq \alpha(t) .
\end{aligned}
$$

Noting that the process $\left(t, S^{t_{m}, S_{m}, i}(t)\right)$ is in $D$, then, by (25), (26) and (27) we find that

$$
\begin{aligned}
& V^{(n)}\left(t_{m}, s_{m}, i\right) \\
& \geq \eta_{m}+E^{\mathbb{Q}}\left[e^{-r\left(\tau \wedge \gamma_{m}-t_{m}\right)} \psi\left(\tau \wedge \gamma_{m}, S\left(\tau \wedge \gamma_{m}\right), i\right) \mid S\left(t_{m}\right)=s_{m}, \alpha\left(t_{m}\right)=i\right] \\
&= \eta_{m}+E^{\mathbb{Q}}\left[e^{-r\left(\tau \wedge \gamma_{m}-t_{m}\right)}\left(\psi(\tau, S(\tau), i) \mathbf{1}_{\left\{\tau<\gamma_{m}\right\}}+\psi\left(\gamma_{m}, S\left(\gamma_{m}\right), i\right) \mathbf{1}_{\left\{\tau \geq \gamma_{m}\right\}}\right) \mid\right. \\
&\left.S\left(t_{m}\right)=s_{m}, \alpha\left(t_{m}\right)=i\right] \\
&= \eta_{m}+E^{\mathbb{Q}}\left[e^{-r\left(\tau \wedge \gamma_{m}-t_{m}\right)}\left(\phi(\tau, S(\tau)) \mathbf{1}_{\left\{\tau<\gamma_{m}\right\}}+\phi\left(\gamma_{m}, S\left(\gamma_{m}\right)\right) \mathbf{1}_{\left\{\tau \geq \gamma_{m}\right\}}\right) \mid\right. \\
&\left.S\left(t_{m}\right)=s_{m}, \alpha\left(t_{m}\right)=i\right] \\
& \geq \eta_{m}+E^{\mathbb{Q}}\left[e ^ { - r ( \tau \wedge \gamma _ { m } - t _ { m } ) } \left(\left(G^{(n)}(\tau, S(\tau), i)+\delta\right) \mathbf{1}_{\left\{\tau<\gamma_{m}\right\}}\right.\right. \\
&\left.\left.+\left(V^{(n)}\left(\gamma_{m}, S\left(\gamma_{m}\right), i\right)+\gamma\right) \mathbf{1}_{\left\{\tau \geq \gamma_{m}\right\}}\right) \mid S\left(t_{m}\right)=s_{m}, \alpha\left(t_{m}\right)=i\right]
\end{aligned}
$$




$$
\begin{aligned}
\geq & \eta_{m}+\delta \wedge \gamma+E^{\mathbb{Q}}\left[e ^ { - r ( \tau \wedge \gamma _ { m } - t _ { m } ) } \left(\left(G^{(n)}(\tau, S(\tau), i)\right) \mathbf{1}_{\left\{\tau<\gamma_{m}\right\}}\right.\right. \\
& \left.\left.+\left(V^{(n)}\left(\gamma_{m}, S\left(\gamma_{m}\right), i\right)\right) \mathbf{1}_{\left\{\tau \geq \gamma_{m}\right\}}\right) \mid S\left(t_{m}\right)=s_{m}, \alpha\left(t_{m}\right)=i\right],
\end{aligned}
$$

since $\eta_{m} \rightarrow 0$ as $m \rightarrow \infty$, and $\tau$ is arbitrary. This provides the contradiction of (24).

\section{Uniqueness of the viscosity solution}

The main task of this section is to prove the comparison principle of the HJB variational inequality problem (7), which ensures the uniqueness of the solution.

Before that, we try to prove the following comparison principle of the HJB equation problem:

$(\mathbb{P}) \begin{cases}F_{1}\left(t, s, \rho_{1}, q, p, M\right):=-q+\left(r+q_{12}\right) \rho_{1}-\mathcal{H}_{1}(t, s, p, M)=0, & (t, s) \in[0, T) \times \mathbb{R}^{+}, \\ F_{2}\left(t, s, \rho_{2}, q, p, M\right):=-q+\left(r+q_{21}\right) \rho_{2}-\mathcal{H}_{2}(t, s, p, M)=0, & (t, s) \in[0, T) \times \mathbb{R}^{+} \\ \rho_{1}(T, s)=\rho_{2}(T, s)=(K-s)^{+}, \quad s \in \mathbb{R}^{+}, & \end{cases}$

where

$$
\mathcal{H}_{i}(t, s, p, M):=-q_{i j} \rho_{j}(t, s)+\beta_{i}\left(\xi_{i}-s\right) p-\frac{\sigma^{2}}{2} M, \quad i \in \mathcal{M}
$$

Since the problem $(\mathbb{P})$ is of the weakly coupled type, we only need to consider the problem

$$
\left(\mathbb{P}^{\prime}\right)\left\{\begin{array}{l}
F_{i}(t, s, W, q, p, M) \\
\quad:=-q+\left(r+q_{i j}\right) W-\mathcal{H}_{i}(t, s, p, M)=0, \quad j \neq i,(t, s) \in[0, T) \times \mathbb{R}^{+} \\
W(T, s)=(K-s)^{+}, \quad s \in \mathbb{R}^{+}
\end{array}\right.
$$

where $V$ is as above. To begin with, we introduce Definitions 5, 6,7 and Ishii's lemma, which will play important roles in the succeeding proof. For more details as regards the above definitions and Ishii's lemma, we refer to the classical literature [21] and [22].

Definition 5 Let $U_{i}$ be upper semicontinous (u.s.c.), $\phi \in C^{1,2}\left([0, T] \times \mathbb{R}^{+}\right)$, and $(\bar{t}, \bar{s}) \in$ $[0, T) \times \mathbb{R}^{+}$be a maximum point of $U_{i}-\phi$. Define a second-order superjet of $U_{i}$ at $(\bar{t}, \bar{s})$ as

$$
\begin{aligned}
\mathcal{P}^{2,+} U_{i}(\bar{t}, \bar{s}):= & \left\{(q, p, M) \in R \times R \times \mathcal{S} \mid U_{i}(t, s) \leq U_{i}(\bar{t}, \bar{s})+q(t-\bar{t})+p(s-\bar{s})\right. \\
& \left.+\frac{1}{2} M(s-\bar{s})^{2}+o\left(|t-\bar{t}|+|s-\bar{s}|^{2}\right)\right\} .
\end{aligned}
$$

Definition 6 Let $V_{i}$ be lower semicontinuous (l.s.c.), $\phi \in C^{1,2}\left([0, T] \times \mathbb{R}^{+}\right)$, and $(\bar{t}, \bar{s}) \in$ $[0, T) \times \mathbb{R}^{+}$be a maximum point of $V_{i}-\phi$. Define a second-order subjet of $V_{i}$ at $(\bar{t}, \bar{s})$ as

$$
\begin{aligned}
\mathcal{P}^{2,-} V_{i}(\bar{t}, \bar{s}):= & \left\{(q, p, M) \in \mathbb{R} \times \mathbb{R} \times \mathcal{S} \mid V_{i}(t, s) \geq V_{i}(\bar{t}, \bar{s})+q(t-\bar{t})+p(s-\bar{s})\right. \\
& \left.+\frac{1}{2} M(s-\bar{s})^{2}+o\left(|t-\bar{t}|+|s-\bar{s}|^{2}\right)\right\} .
\end{aligned}
$$


Definition 7 An u.s.c. (resp. l.s.c.) function $w_{i}$ on $[0, T) \times \mathbb{R}^{+}$is a viscosity subsolution (resp. supersolution) of $(\mathbb{P})$ on $[0, T) \times \mathbb{R}^{+}$if and only if for all $(t, s) \in[0, T) \times \mathbb{R}^{+}$and all $(q, p, M) \in \mathcal{P}^{2,+} w(t, s)\left(\operatorname{resp} . \mathcal{P}^{2,-} w_{i}(t, s)\right)$,

$$
F\left(t, s, w_{i}(t, s), q, p, M\right) \leq 0 \quad(\text { resp. } \geq 0) .
$$

Lemma 6 (Ishii's lemma) Let $U_{i}\left(V_{i}\right)$ be a u.s.c. (l.s.c.) function on $[0, T) \times \mathbb{R}^{+}, \varphi \in$ $C^{1,1,2,2}\left([0, T)^{2} \times \mathbb{R}^{+} \times \mathbb{R}^{+}\right)$and $(\bar{t}, \bar{\tau}, \bar{x}, \bar{y}) \in[0, T)^{2} \times \mathbb{R}^{+} \times \mathbb{R}^{+}$a local maximum (minimum) of $U_{i}(t, x)-V_{i}(\tau, y)-\varphi(t, \tau, x, y)$. Then, for all $\eta>0$, there exist $M, N \in \mathcal{S}$ satisfying

$$
\begin{aligned}
& \left(\frac{\partial \varphi}{\partial t}(\bar{t}, \bar{\tau}, \bar{x}, \bar{y}), D_{x} \varphi(\bar{t}, \bar{\tau}, \bar{x}, \bar{y}), M\right) \in \mathcal{P}^{2,+} U_{i}(\bar{t}, \bar{x}), \\
& \left(-\frac{\partial \varphi}{\partial \tau}(\bar{t}, \bar{\tau}, \bar{x}, \bar{y}),-D_{y} \varphi(\bar{t}, \bar{\tau}, \bar{x}, \bar{y}), N\right) \in \mathcal{P}^{2,-} V_{i}(\bar{\tau}, \bar{y}),
\end{aligned}
$$

and

$$
\left(\begin{array}{cc}
M & 0 \\
0 & -N
\end{array}\right) \leq D_{x y}^{2} \varphi(\bar{t}, \bar{\tau}, \bar{x}, \bar{y})+\eta\left(D_{x y}^{2} \varphi(\bar{t}, \bar{\tau}, \bar{x}, \bar{y})\right)^{2} .
$$

Now we proceed to the statement of the comparison principle for the problem $\left(\mathbb{P}^{\prime}\right)$, and the proof of it.

Theorem 3 Let $U_{i}$ be a u.s.c. viscosity subsolution of problem $\left(\mathbb{P}^{\prime}\right)$ with polynomial growth condition, and let $V_{i}$ be l.s.c. viscosity supersolution of problem $\left(\mathbb{P}^{\prime}\right)$ with polynomial growth condition. In addition, let $U_{i}(T, s)=V_{i}(T, s)=(K-s)^{+}$for $s \in \mathbb{R}^{+}$. If $U_{i}(T, \cdot) \leq V_{i}(T, \cdot)$, then $U_{i} \leq V_{i}$ in $[0, T) \times \mathbb{R}^{+}$.

Proof We argue by contradiction.

Step 1 . Assume that the supreme of $U_{i}-V_{i}$ on $[0, T] \times \mathbb{R}^{+}$is attained at $(\bar{t}, \bar{x}) \in[0, T) \times \mathcal{O}$ for some bounded set $\mathcal{O} \subset \mathbb{R}^{+}$, i.e.,

$$
M:=\sup _{[0, T] \times \mathbb{R}^{+}}\left(U_{i}-V_{i}\right)=\max _{[0, T) \times \mathcal{O}}\left(U_{i}-V_{i}\right)=\left(U_{i}-V_{i}\right)(\bar{t}, \bar{x}),
$$

and suppose that

$$
M>0 \text {. }
$$

Then, for any $\epsilon>0$, we define

$$
\Phi_{\epsilon}(t, \tau, x, y)=U_{i}(t, x)-V_{i}(\tau, y)-\varphi_{\epsilon}\left(t_{\epsilon}, \tau_{\epsilon}, x_{\epsilon}, y_{\epsilon}\right)
$$

with

$$
\varphi_{\epsilon}(t, \tau, x, y)=\frac{1}{2 \epsilon}\left[(t-\tau)^{2}+(x-y)^{2}\right] \geq 0 .
$$


Similar to (29), we denote

$$
M_{\epsilon}:=\sup _{[0, T]^{2} \times \mathcal{O}^{2}} \Phi_{\epsilon}=U_{i}\left(t_{\epsilon}, x_{\epsilon}\right)-V_{i}\left(\tau_{\epsilon}, y_{\epsilon}\right)-\varphi_{\epsilon}\left(t_{\epsilon}, \tau_{\epsilon}, x_{\epsilon}, y_{\epsilon}\right)
$$

In the sequel, we try to show

$$
M_{\epsilon} \rightarrow M, \quad \varphi_{\epsilon} \rightarrow 0, \quad \text { as } \epsilon \rightarrow 0 .
$$

Note that

$$
M \leq M_{\epsilon},
$$

which implies that

$$
\left(U_{i}-V_{i}\right)(\bar{t}, \bar{x}) \leq U_{i}\left(t_{\epsilon}, x_{\epsilon}\right)-V_{i}\left(\tau_{\epsilon}, y_{\epsilon}\right)-\varphi_{\epsilon}\left(t_{\epsilon}, \tau_{\epsilon}, x_{\epsilon}, y_{\epsilon}\right)
$$

i.e.,

$$
\varphi_{\epsilon}\left(t_{\epsilon}, \tau_{\epsilon}, x_{\epsilon}, y_{\epsilon}\right) \leq U_{i}\left(t_{\epsilon}, x_{\epsilon}\right)-V_{i}\left(\tau_{\epsilon}, y_{\epsilon}\right)-\left(U_{i}-V_{i}\right)\left(t^{*}, s^{*}\right)
$$

which indicates that $\varphi_{\epsilon}\left(t_{\epsilon}, \tau_{\epsilon}, x_{\epsilon}, y_{\epsilon}\right)$ is bounded. Then sending $\epsilon \rightarrow 0^{+}$, we find from (31) and (34) that

$$
\left(t_{\epsilon}, \tau_{\epsilon}, x_{\epsilon}, y_{\epsilon}\right) \rightarrow(\bar{t}, \bar{\tau}, \bar{x}, \bar{y}) \in[0, T]^{2} \times \mathcal{O}^{2} \quad \text { and } \quad \bar{t}=\bar{\tau}, \quad \bar{x}=\bar{y}
$$

Hence,

$$
0 \leq \lim _{\epsilon \rightarrow 0} \varphi_{\epsilon}\left(t_{\epsilon}, \tau_{\epsilon}, x_{\epsilon}, y_{\epsilon}\right) \leq\left(U_{i}-V_{i}\right)(\bar{t}, \bar{x})-(U-V)(\bar{t}, \bar{x}) \leq 0
$$

which implies $\varphi_{\epsilon} \rightarrow 0$ as $\epsilon \rightarrow 0$. From (32), (35), (36), $M_{\epsilon} \rightarrow M$.

In view of Ishii's lemma, there exist $M, N \in \mathcal{S}$ satisfying

$$
\left(\begin{array}{cc}
M & 0 \\
0 & -N
\end{array}\right) \leq \frac{3}{\epsilon}\left(\begin{array}{cc}
1 & -1 \\
-1 & 1
\end{array}\right),
$$

which implies that

$$
\sigma^{2}\left(x_{\epsilon}\right) M-\sigma^{2}\left(y_{\epsilon}\right) N \leq \frac{3}{\epsilon}\left|\sigma^{2}\left(x_{\epsilon}\right)-\sigma^{2}\left(y_{\epsilon}\right)\right|^{2}=0 \quad\left(\text { since } \sigma^{2}\left(x_{\epsilon}\right)=\sigma^{2}\left(y_{\epsilon}\right)\right),
$$

and

$$
\begin{aligned}
& \left(\frac{1}{\epsilon}\left(t_{\epsilon}-\tau_{\epsilon}\right), \frac{1}{\epsilon}\left(x_{\epsilon}-y_{\epsilon}\right), M\right) \in \mathcal{P}^{2,+} U\left(t_{\epsilon}, x_{\epsilon}\right), \\
& \left(\frac{1}{\epsilon}\left(t_{\epsilon}-\tau_{\epsilon}\right), \frac{1}{\epsilon}\left(x_{\epsilon}-y_{\epsilon}\right), N\right) \in \mathcal{P}^{2,-} V\left(\tau_{\epsilon}, y_{\epsilon}\right) .
\end{aligned}
$$


Step 2. Since $U_{i}, V_{i}$ are, respectively, a subsolution and a supersolution of $\left(\mathbb{P}^{\prime}\right)$, we have

$$
\begin{aligned}
& -\frac{1}{\epsilon}\left(t_{\epsilon}-\tau_{\epsilon}\right)+\left(r+q_{i j}\right) U_{i}\left(t_{\epsilon}, x_{\epsilon}\right)-\mathcal{H}_{i}\left(t_{\epsilon}, x_{\epsilon}, \frac{1}{\epsilon}\left(x_{\epsilon}-y_{\epsilon}\right), M\right) \leq 0, \\
& -\frac{1}{\epsilon}\left(t_{\epsilon}-\tau_{\epsilon}\right)+\left(r+q_{i j}\right) V_{i}\left(\tau_{\epsilon}, y_{\epsilon}\right)-\mathcal{H}_{i}\left(\tau_{\epsilon}, y_{\epsilon}, \frac{1}{\epsilon}\left(x_{\epsilon}-y_{\epsilon}\right), N\right) \geq 0,
\end{aligned}
$$

which leads to

$$
\begin{aligned}
(r & \left.+q_{i j}\right)\left(U_{i}\left(t_{\epsilon}, x_{\epsilon}\right)-V_{i}\left(\tau_{\epsilon}, y_{\epsilon}\right)\right) \\
& \leq \mathcal{H}_{i}\left(t_{\epsilon}, x_{\epsilon}, \frac{1}{\epsilon}\left(x_{\epsilon}-y_{\epsilon}\right), M\right)-\mathcal{H}_{i}\left(\tau_{\epsilon}, y_{\epsilon}, \frac{1}{\epsilon}\left(x_{\epsilon}-y_{\epsilon}\right), N\right) \\
& \leq q_{i j}\left(V_{j}\left(\tau_{\epsilon}, y_{\epsilon}\right)-U_{j}\left(t_{\epsilon}, x_{\epsilon}\right)\right)-\frac{1}{\epsilon}\left(x_{\epsilon}-y_{\epsilon}\right)^{2}+\frac{\sigma^{2}}{2}(M-N) \\
& \leq q_{i j} C\left(\left|t_{\epsilon}-\tau_{\epsilon}\right|^{\frac{1}{2}}+\left|x_{\epsilon}-y_{\epsilon}\right|\right)-\frac{1}{\epsilon}\left|x_{\epsilon}-y_{\epsilon}\right|^{2} .
\end{aligned}
$$

Finally, after sending $\epsilon$ to 0 , we conclude that

$$
\left(r+q_{i j}\right) M=\left(r+q_{i j}\right)\left(U_{i}(\bar{t}, \bar{x})-V_{i}(\bar{\tau}, \bar{y})\right) \leq 0,
$$

which contradicts the assumption (30).

Now we consider the corresponding coupled variational inequalities problem,

$$
(\mathbb{C}) \begin{cases}\min \left\{F_{1}\left(t, s, \rho_{1}, q, p, M\right), \rho_{1}-G_{1}^{(n)}\right\}=0, & (t, s) \in[0, T) \times \mathbb{R}^{+}, \\ \min \left\{F_{2}\left(t, s, \rho_{2}, q, p, M\right), \rho_{2}-G_{2}^{(n)}\right\}=0, & (t, s) \in[0, T) \times \mathbb{R}^{+}, \\ \rho_{1}(T, s)=\rho_{2}(T, s)=(K-s)^{+}, \quad s \in \mathbb{R}^{+} . & \end{cases}
$$

Similarly, we only need to consider the problem

$$
(\mathbb{U N C})\left\{\begin{array}{l}
\min \left\{F_{i}(t, s, W, q, p, M), W-G_{i}^{(n)}\right\}=0, \quad j \neq i,(t, s) \in[0, T) \times \mathbb{R}^{+}, \\
W(T, s)=(K-s)^{+}, \quad s \in \mathbb{R}^{+} .
\end{array}\right.
$$

Theorem 4 Let $U_{i}$ be a u.s.c. viscosity subsolution of problem $(\mathbb{U N C})$ with polynomial growth condition, and let $V_{i}$ be l.s.c. viscosity supersolution of problem $(\mathbb{U N C})$ with polynomial growth condition. If $U_{i}(T, \cdot) \leq V_{i}(T, \cdot)$, then $U_{i} \leq V_{i}$ in $[0, T) \times \mathbb{R}^{+}$.

Proof The only difference appears at Step 2 in the proof of Theorem 3, that is,

$$
\begin{aligned}
& \min \left\{F_{i}\left(t_{\epsilon}, x_{\epsilon}, W, q, p, M\right), U_{i}-G_{i}^{(n)}\right\} \leq 0, \\
& \min \left\{F_{i}\left(\tau_{\epsilon}, y_{\epsilon}, W, q, p, M\right), V_{i}-G_{i}^{(n)}\right\} \geq 0 .
\end{aligned}
$$

These two inequalities lead to the following two cases:

1 Either $U_{i}-G_{i}^{(n)} \leq 0$. Together with the inequality $V_{i}-G_{i}^{(n)} \geq 0$ leads to contradiction of (29). 
2 Or $F_{i}\left(t_{\epsilon}, x_{\epsilon}, W, q, p, M\right) \leq 0$, which can be combined with the supersolution part $F_{i}\left(\tau_{\epsilon}, y_{\epsilon}, W, q, p, M\right) \geq 0$ exactly as in the proof of Theorem 3 , and this leads to the same contradiction as above.

\section{Acknowledgements}

Not applicable.

\section{Funding}

This work was supported by the Fundamental Research Funds for the Central Universities (nos. JBK1803014, JBK170940, and JBK151126).

\section{Availability of data and materials}

Not applicable.

\section{Competing interests}

The authors declare that they have no competing interests.

\section{Authors' contributions}

The manuscript was written through contributions of all authors. All authors read and approved the final manuscript.

\section{Publisher's Note}

Springer Nature remains neutral with regard to jurisdictional claims in published maps and institutional affiliations.

Received: 31 August 2018 Accepted: 9 November 2018 Published online: 16 November 2018

\section{References}

1. Bierbrauer, M., Trück, S., Weron, R.: Modeling electricity prices with regime switching models. Int. Conf. Comput. Sci. 3039, 859-867 (2005)

2. De Jong, C.: The nature of power spikes: a regime-switch approach. Stud. Nonlinear Dyn. Econom. (2005) https://doi.org/10.2202/1558-3708.1361

3. Hirsch, G.: Pricing of hourly exercisable electricity swing options using different price processes. J. Energy Mark. 2, 2 (2009)

4. Janczura, J., Weron, R.: An empirical comparison of alternate regime-switching models for electricity spot prices Energy Econ. 32(5), 1059-1073 (2010)

5. Janczura, J., Weron, R.: Goodness-of-fit testing for the marginal distribution of regime-switching models with an application to electricity spot prices. AStA Adv. Stat. Anal. 97(3), 239-270 (2013)

6. Wahab, M.I.M., Yin, Z., Edirisinghe, N.C.P.: Pricing swing options in the electricity markets under regime-switching uncertainty. Quant. Finance 10(9), 975-994 (2010)

7. Wahab, M.I.M., Lee, C.: Pricing swing options with regime switching. Ann. Oper. Res. 185(1), 139-160 (2011)

8. Chiarella, C., Clewlow, L., Kang, B.: The evaluation of multiple year gas sales agrement with regime switching. Int. J. Theor. Appl. Finance, 19(01), 1-25 (2016)

9. Bauerle, N., Riess, V.: Gas storage valuation with regime switching. Energy Syst. 7(3), 499-528 (2016)

10. Dahlgren, M.: A continuous time model to price commodity-based swing options. Rev. Deriv. Res. 8(1), 27-47 (2005)

11. Dahlgren, M., Korn, R.: The swing option on the stock market. Int. J. Theor. Appl. Finance 08(01), 123-139 (2005)

12. Carmona, R., Touzi, N.: Optimal multiple stopping and valuation of swing options. Math. Finance 18(2), 239-268 (2008)

13. Wilhelm, M., Winter, C.: Finite element valuation of swing options. J. Comput. Finance 11(3), 107-132 (2008)

14. Touzi, N.: Optimal Stochastic Control, Stochastic Target Problems, and Backward SDE. Springer, New York (2013)

15. Fleming, W.H., Soner, H.M.: Controlled Markov Processes and Viscosity Solutions. Springer, New York (2006)

16. Basei, M., Cesaroni, A., Vargiolu, T.: Optimal exercise of swing contracts in energy markets: an integral constrained stochastic optimal control problem. SIAM J. Financ. Math. 5(1), 581-608 (2014)

17. Ben Latifa, I., Bonnans, J.F., Mnif, M.: A general optimal multiple stopping problem with an application to swing options. Stoch. Anal. Appl. 33(4), 715-739 (2015)

18. Weron, R.: Modeling and Forecasting Electricity Loads and Prices: A Statistical Approach. Wiley, Chichester (2006)

19. Karatzas, I., Shreve, S.E.: Brownian Motion and Stochastic Calculus, 2nd edn. Springer, New York (1991)

20. Pemy, M., Zhang, Q.: Optimal stock liquidation in a regime switching model with finite time horizon. J. Math. Anal. Appl. 321(2), 537-552 (2006)

21. Ishii, H.: The maximum principle for semicontinuous functions. Differ. Integral Equ. 3(6), 1001-1014 (1990)

22. Crandall, M.G., Ishii, H., Lions, P.L.: User's guide to viscosity solutions of second order partial differential equations. Bull. Am. Math. Soc. 27(1), 1-67 (1992) 